\title{
Credit Risk Model with Contagious Default Dependencies Affected by Macro-Economic Condition
}

\author{
Hideyuki Takada ${ }^{\dagger}$ and Ushio Sumita ${ }^{\ddagger}$ \\ ${ }^{\dagger}$ Mizuho-DL Financial Technology Co., Ltd, Chiyoda-ku 100-0004, Japan \\ ${ }^{\ddagger}$ Graduate School of Systems and Information Engineering, University of Tsukuba, \\ Tsukuba-City 305-8573, Japan
}

May 11, 2010

\begin{abstract}
We consider a credit risk model with two industrial sectors, where defaults of corporations would be influenced by two factors. The first factor represents the macro economic condition which would affect the default intensities of the two industrial sectors differently. The second factor reflects the influences of the past defaults of corporations against other active corporations, where such influences would affect the two industrial sectors differently. A two-layer Markov chain model is developed, where the macro economic condition is described as a birth-death process, while another Markov chain represents the stochastic characteristics of defaults with default intensities dependent on the state of the birth-death process and the number of defaults in two sectors. Although the state space of the two-layer Markov chain is huge, the fundamental absorbing process with a reasonable state space size could capture the first passage time structure of the two-layer Markov chain, thereby enabling one to evaluate the joint probability of the number of defaults in two sectors via the uniformization procedure of Keilson. This in turn enables one to value a variety of derivatives defined on the underlying credit portfolios. In this paper, we focus on a financial product called CDO, and a related option.
\end{abstract}

\section{Introduction}

It is often observed that the corporate defaults at the time of a recession tend to cluster in a relatively short time period. This clustering phenomenon may result from the complex business interactions among many corporations affected simultaneously by the recession. One of the most prevalent approaches to cope with such business interactions would be a doubly stochastic model, where corporations are related through their exposure to common risk factors, and the co-movements of such factors induce the correlated corporate defaults. However, the doubly stochastic model approach assumes that, given a state of the exogenous process describing the macro-economic condition, individual corporate defaults occur independently. Some empirical studies such as Das et al.(2007) and Azizpour and Giesecke(2008), for example, report that the doubly stochastic approach is inadequate so as to capture the default clustering, and suggest the importance of introducing the feedback mechanism from default events. Collin-Dufresne et al.(2003) also claim that default events are often accompanied by significant increases of the credit spreads of corporate bonds and CDSs of other corporations. Accordingly, in the credit risk management and derivatives pricing, it is important to cope with two different factors affecting corporate defaults simultaneously: the macro-economic condition and the default contagion or the default feedback effect.

In order to cope with this challenge, many different models have been proposed in the literature. Jarrow and $\mathrm{Yu}(2001)$ investigate the pricing of risky debt in the primary-secondary framework, where the default intensity of primary corporations depend only on the macro-economic condition, while that of the secondary corporations depend on both the macro-economic condition and the number of the primary corporation defaulted by the present time. Yu (2007) extends this paper by introducing the concept of the total hazard construction, where the default intensities of corporations are affected by common exogenous factors as well as the number of defaulted corporations in the portfolio. Frey and Backhaus(2006) apply Markov process techniques to construct and analyze 
interacting default intensities, where the default intensities of active corporations are exogenously specified and the dependence structure of the default times is endogenously determined. They also use Monte Carlo simulation for pricing multi-name credit derivatives. Using the matrix-analytic method, a closed form pricing formula for multi-name credit derivatives is given by Herbertsson and Rootzen(2008), where default intensities are assumed to be constant between defaults but that can jump at the times of default. The model, however, could tolerate only a rather small number of corporations, say 15, because of the computational burden involved. Zeheng and Jiang(2009) deal with multi-name credit derivatives pricing by developing a factor contagion model having the heterogeneous conditionally independent portfolio and infectious default portfolio as special cases. This model assumes that the default intensities are driven by external common factors as well as defaults of other corporations in the portfolio. They suggest that the analytical formula for pricing multi name credit derivatives can be derived via total hazard construction combined with the recursive method.

In comparison with the literature discussed above, this paper proposes two new features. The first feature is that two industrial sectors are incorporated explicitly, where the default contagion within the same industrial sector and that from the other industrial sector are treated in a distinguishable manner. The second feature is to develop two-layer Markov chain model, where the default phenomenon can be described in terms of a sequence of matrix convolutions, thereby enabling one to deal with the large number of corporations in each industrial sector. Combined with the uniformization procedure of Keilson(1979), the time dependent joint probability of the number of defaulted corporations in one industrial sector and that in the other industrial sector can be computed with speed and accuracy, totally eliminating the necessity of Monte Caro simulation.

We consider a credit risk model with two industrial sectors $\mathcal{A}$ and $\mathcal{B}$ with $M_{\mathcal{A}}$ and $M_{\mathcal{B}}$ active corporations respectively at time $t=0$, where defaults of corporations would be influenced by two factors. The first factor represents the changes of the macro economic condition which would affect the default intensities of the two industrial sectors differently. The second factor reflects the influences of the past defaults of corporations against other active corporations, where such influences would affect the two industrial sectors differently as for the macro economic factor. The effect of the macro-economic condition to one industrial sector can also be different from that to the other industrial sector, although all corporations in each industrial sector are assumed to be affected uniformly. In order to incorporate such interactions between the macro economic condition and defaults as well as the contagion phenomenon among the defaults, a two-layer Markov chain model is developed, where the macro economic condition is described as a birth-death process, while another Markov chain represents the stochastic characteristics of defaults with default intensities dependent on the state of the birth-death process, the number of defaults in $\mathcal{A}$ and that in $\mathcal{B}$ by time $t$. Although the state space of the two-layer Markov chain is huge, the fundamental absorbing process with a resonable state space size could capture the first passage time structure of the twolayer Markov chain, thereby enabling one to evaluate the joint probability of the number of defaults in $\mathcal{A}$ and that in $\mathcal{B}$ by time $t$ via the uniformization procedure of Keilson with speed and accuracy. This in turn enables one to value a variety of derivatives defined on the underlying credit portfolios. In this paper, we focus on a financial product called " Collateralized Debt Obligation (CDO) " , and a related CDO option of European type.

CDO is a structured product that securitizes a reference portfolio of default risky instruments such as loans or bonds or CDSs. A credit default swap offers protection against default of a certain underlying entity over specified time horizon. In the CDO scheme, given a reference portfolio, the associated credit risk is divided into tranches of increasing seniority, where a tranche is defined by a pair of an attachment point and a detachment point of the cumulative aggregate loss of the reference portfolio. Here, the attachment point $K_{a}$ means that the protection buyer (the CDO issuer) is fully responsible for the portfolio loss up to $K_{a}$. On the other hand, the protection seller (the tranche investor) compensates the portfolio loss beyond $K_{a}$ up to $K_{d}$ for the protection buyer, where $K_{d}$ is the detachment point. Predetermined premiums are paid to the protection seller by 
the protection buyer according to a predetermined schedule up to the maturity year in such a way that no-arbitrage condition of the credit derivatives market is satisfied.

The principal entity of interest is a reference portfolio consisting of $M_{\mathcal{A}}$ corporations in an industrial sector $\mathcal{A}$ and $M_{\mathcal{B}}$ corporations in another industrial sector $\mathcal{B}$. As time progresses, some of them would default. Let $D_{\mathcal{A}}(t)$ and $D_{\mathcal{B}}(t)$ be the number of defaulted corporations in $\mathcal{A}$ by time $t$ and that in $\mathcal{B}$ respectively. It is assumed that each default in $\mathcal{A}$ results in the bad debt of $B D_{\mathcal{A}}$, which is called the notional amount. It is also assumed that the recovery rate of the bad debt for a default in $\mathcal{A}$ is given by $R_{\mathcal{A}}$. We deffine $B D_{\mathcal{B}}$ and $R_{\mathcal{B}}$ similarly. The cumulative loss up to time $t$ denoted by $l(t)$ is then given by

$$
l(t)=B D_{\mathcal{A}} \cdot\left(1-R_{\mathcal{A}}\right) \cdot D_{\mathcal{A}}(t)+B D_{\mathcal{B}} \cdot\left(1-R_{\mathcal{B}}\right) \cdot D_{\mathcal{B}}(t) .
$$

For analytical convienence, we also define $M_{\mathcal{A}}(t)$ and $M_{\mathcal{B}}(t)$ as the number of corporations in $\mathcal{A}$ still active at time $t$ and that in $\mathcal{B}$ respectively, i.e.,

$$
M_{\mathcal{A}}(t)=M_{\mathcal{A}}-D_{\mathcal{A}}(t) ; M_{\mathcal{B}}(t)=M_{\mathcal{B}}-D_{\mathcal{B}}(t) .
$$

We now turn our attention to the procedural details of the CDO contract characterized by an attachment point $K_{a}$, a detachment point $K_{d}, \underline{\tau}=\left(\tau_{0}, \cdots, \tau_{K}\right)$ specifying the sequence of time epochs at which the contract would be exercised with $\tau_{0}=0$, and the contruct premium $c_{\left[K_{a}, K_{d}\right]}$. The protection seller taking the credit exposure to the tranche with $K_{a}$ and $K_{d}$ will bear full losses occurring in the portfolio in excess of $K_{a}$ but up to $K_{d}$. Such a tranche is denoted by $\left[K_{a}, K_{d}\right]$ Tranche. Let $L_{\left[K_{a}, K_{d}\right]}(t)$ be the cumulative amount paid by the protection seller to the protection buyer by time $t$. One then sees that

$$
L_{\left[K_{a}, K_{d}\right]}(t)=\left\{\begin{array}{ll}
0 & \text { if } l(t) \leq K_{a} \\
l(t)-K_{a} & \text { if } K_{a} \leq l(t) \leq K_{d} \\
K_{d}-K_{a} & \text { if } K_{d} \leq l(t)
\end{array} .\right.
$$

At time $\tau_{k}\left(k=1, \cdots, K\right.$ with $\left.\tau_{0}=0\right)$, the protection seller pays to the protection buyer by the amount of $P A Y_{\text {sell } \rightarrow \text { buy }}\left(\tau_{k}\right)$ specified by the tranched loss increment, i.e.

$$
P A Y_{\text {sell } \rightarrow \text { buy }}\left(\tau_{k}\right)=L_{\left[K_{a}, K_{d}\right]}\left(\tau_{k}\right)-L_{\left[K_{a}, K_{d}\right]}\left(\tau_{k-1}\right), \quad k=1,2, \cdots, K .
$$

It should be noted from (1.3) that once the cumulative payment from the protection seller to the protection buyer reaches $K_{d}-K_{a}, P A Y_{\text {sell } \rightarrow \text { buy }}$ remains to be 0 from that point on. In exchange, a payment would be made from the protection buyer to the protection seller at each time epoch $\tau_{k}(k=0,1, \cdots, K)$. This payment amount, denoted by $P A Y_{b u y \rightarrow \text { sell }}$, is based on the unit premium $c_{\left[K_{a}, K_{d}\right]}$ agreed upon at time $\tau_{0}=0$, which is applied to the hedge interval, i.e. $\left(K_{d}-K_{a}\right)$ minus the cumulative payment made by the protection seller to the protection buyer up to time $\tau_{k}$. More formally, one has

$$
P A Y_{\text {buy } \rightarrow \text { sell }}\left(c_{\left[K_{a}, K_{d}\right]}, \tau_{k}\right)=c_{\left[K_{a}, K_{d}\right]}\left(\left(K_{d}-K_{a}\right)-\sum_{j=1}^{k} P A Y_{\text {sell } \rightarrow \text { buy }}\left(\tau_{j}\right)\right) .
$$

From (1.4), this then leads to

$$
P A Y_{\text {buy } \rightarrow \text { sell }}\left(c_{\left[K_{a}, K_{d}\right]}, \tau_{k}\right)=c_{\left[K_{a}, K_{d}\right]}\left(\left(K_{d}-K_{a}\right)-L_{\left[K_{a}, K_{d}\right]}\left(\tau_{k}\right)\right) .
$$

The financial interactions between the protection seller and the protection buyer are illustrated in Figure 1.1.

In formulating $\mathrm{CDO}$ contracts, the key question is how to determine the unit premium $c_{\left[K_{a}, K_{d}\right]}^{*}$ at equilibrium which assures no-arbitrage in the credit derivatives market. We assume that there 


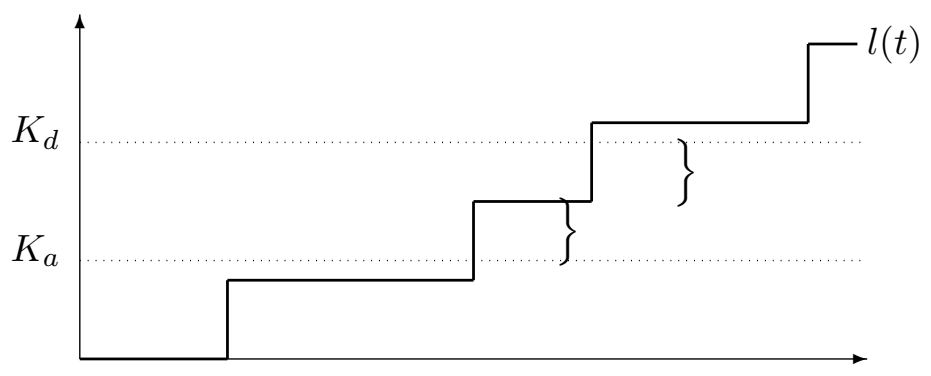

Protection Seller

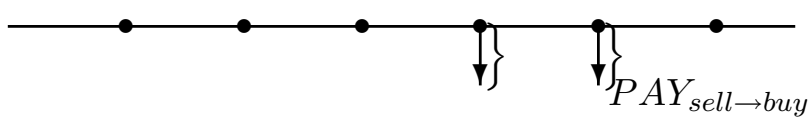

Protection Buyer

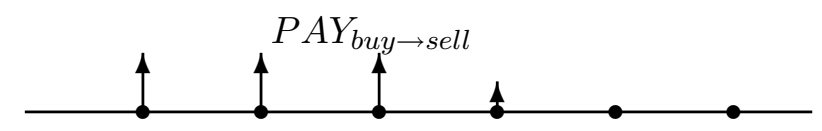

Figure 1.1: Protectionleg and Premiumleg

exists a risk-neutral martingale measure $\mathbb{P}$ under which all price processes discounted with the interest rate $r$ are martingales. Furthermore, it is assumed that $r$ is deterministic. In this paper, all expectations are taken with respect to this measure. Then the unit premium $c_{\left[K_{a}, K_{d}\right]}^{*}$ should satisfy

$$
\sum_{k=1}^{K} e^{-r \tau_{k}} \mathbb{E}\left[P A Y_{\text {sell } \rightarrow \text { buy }}\left(\tau_{k}\right)\right]=\sum_{k=0}^{K-1} e^{-r \tau_{k}} \mathbb{E}\left[P A Y_{\text {buy } \rightarrow \operatorname{sell}}\left(c_{\left[K_{a}, K_{d}\right]}^{*}, \tau_{k}\right)\right]
$$

The left hand side of Equation (1.6) is called the protectionleg while the right hand side is called the premiumleg. From (1.4) and (1.5), Equation (1.6) can be solved for $c_{\left[K_{a}, K_{d}\right]}^{*}$ as

$$
c_{\left[K_{a}, K_{d}\right]}^{*}=\frac{\sum_{k=1}^{K} e^{-r \tau_{k}} \mathbb{E}\left[L_{\left[K_{a}, K_{d}\right]}\left(\tau_{k}\right)-L_{\left[K_{a}, K_{d}\right]}\left(\tau_{k-1}\right)\right]}{\sum_{k=0}^{K-1} e^{-r \tau_{k}} \mathbb{E}\left[\left(K_{d}-K_{a}\right)-L_{\left[K_{a}, K_{d}\right]}\left(\tau_{k}\right)\right]} .
$$

Following [11] and [8], we next consider a CDO contract possibly to be entered at a future point $\tau_{0}=t_{0}$ with option of not exercising it. More formally, an option is offered to the protection buyer concerning whether or not to enter a CDO contract with a tranche $\left[K_{a}, K_{d}\right]$, the contract epoch vector $\underline{\tau}=\left(\tau_{0}, \cdots, \tau_{K}\right)$ to be commenced at $\tau_{0}=t_{0}>0$ and the predetermined contract premium $\tilde{c}_{\left[K_{a}, K_{d}\right]}$. In order to evaluate the value of this option at time 0 , we introduce the Mark-to-Market value for the protection buyer at time $\tau_{0}$, denoted by $\operatorname{Mt} M\left(\tau_{0}\right)$, which is given by

$$
\operatorname{MtM}\left(\tau_{0}\right)=\sum_{k=0}^{K} e^{-r \tau_{k}} \mathbb{E}\left[P A Y_{\text {sell } \rightarrow \text { buy }}\left(\tau_{k}\right)\right]-\sum_{k=0}^{K} e^{-r \tau_{k}} \mathbb{E}\left[P A Y_{\text {buy } \rightarrow \text { sell }}\left(\tilde{c}_{\left[K_{a}, K_{d}\right]}, \tau_{k}\right)\right]
$$

This future value is generally uncertain because the market premium at time $\tau_{0}$ will differ from the contract premium $\tilde{c}_{\left[K_{a}, K_{d}\right]}$, due to changes in the market's view of the expectation of succesive deafaults. One can see that the option value at time 0 for the protection buyer is given by

$$
e^{-r \tau_{0}} \mathbb{E}\left[\max \left\{M t M\left(\tau_{0}\right), 0\right\}\right] .
$$

Similarly, the same option can be offered to the protection seller, who would value the option at time 0 as

$$
e^{-r \tau_{0}} \mathbb{E}\left[\max \left\{-M t M\left(\tau_{0}\right), 0\right\}\right]
$$


The two-layer Markov chain model proposed in this paper enables one to see the impact of contagion effect by developing efficient computational algorithms for evaluating the risk neutral CDO contract premium $c_{\left[K_{a}, K_{d}\right]}^{*}$ based on (1.7) and the option values in (1.9) and (1.10), when both the macro economic factor and the effects of default interactions across two industrial sectors are present.

This paper consists of six sections apart from this introduction section. In Section 2, the twolayer Markov chain model is formally introduced for capturing the effects of the macro economic condition on individual defaults, as well as the impact of defaults on performance of active corporations. Section 3 is devoted to dynamic analysis of the fundamental absorbing trivariate process and its first passage time structure. The joint probability of the number of defaults in $\mathcal{A}$ and that in $\mathcal{B}$ by time $t$ is analyzed explicitly in Section 4, and the computational algorithms for evaluating the joint probability are developed in Section 5. Evaluation of CDO premiums and prices of CDO option of European type is discussed in Section 6, and numerical examples are presented in Section 7. Finally, some concluding remarks are given in Section 8.

\section{Model Description}

Let $\{J(t): t \geq 0\}$ be a Birth-Death process on $\mathcal{J}=\{0,1, \cdots, J\}$, describing the macro economic condition at time $t$, governed by upward transition rates $\nu_{i}^{+},(i=0,1, \cdots, J-1)$ and downward transition rates $\nu_{i}^{-},(i=1, \cdots, J)$. When $J(t)=i \in \mathcal{J}$, individual active corporations in $\mathcal{A}$ and $\mathcal{B}$ would have the default intensities $\xi_{\mathcal{A}}(i)$ and $\xi_{\mathcal{B}}(i)$ respectively. In addition, at the time of a state change of $J(t)$, at most one active corporation in $\mathcal{A}$ or $\mathcal{B}$ may default instantaneously with certain probability, but never both at a time. A default occurs with probability $1-\Theta_{i j}$, and $\Theta_{i j}^{\mathcal{A}}$ and $\Theta_{i j}^{\mathcal{B}}$ represent the probability of this default to occur in $\mathcal{A}$ and that to occur in $\mathcal{B}$ respectively. Accordingly, one has $0 \leq \Theta_{i j}, \Theta_{i j}^{\mathcal{A}}, \Theta_{i j}^{\mathcal{B}} \leq 1$ and $\Theta_{i j}+\Theta_{i j}^{\mathcal{A}}+\Theta_{i j}^{\mathcal{B}}=1$. For the convenience of later references, we summarize:

\section{Definition 2.1}

(a) $\xi_{\mathcal{A}}(i)\left[\xi_{\mathcal{B}}(i)\right]:$ the default intensity of an active corporation in $\mathcal{A}[\mathcal{B}]$ triggered by the macroeconomic factor when $J(t)=i$.

(b) $\Theta_{i j}$ : the probability of having no defaults upon occurrence of a transition of $J(t)$ from $i$ to $j$.

(c) $\Theta_{i j}^{\mathcal{A}}\left[\Theta_{i j}^{\mathcal{B}}\right]$ : the probability of having a default in $\mathcal{A}[\mathcal{B}]$ upon occurrence of a transition of $J(t)$ from $i$ to $j$.

As for the second factor reflecting the influences of the past defaults against other active corporations, let $M_{\mathcal{A}}(t)$ be the stochastic process on $\mathcal{M}_{\mathcal{A}}=\left\{0,1, \cdots, M_{\mathcal{A}}\right\}$ describing the number of active corporations in $\mathcal{A}$ at time $t$, and define $M_{\mathcal{B}}(t)$ on $\mathcal{M}_{\mathcal{B}}=\left\{0,1, \cdots, M_{\mathcal{B}}\right\}$ similarly. For notational convenience, we write $\underline{M}(t)=\left[M_{\mathcal{A}}(t), M_{\mathcal{B}}(t)\right]$ with its state space $\mathcal{M}=\mathcal{M}_{\mathcal{A}} \times \mathcal{M}_{\mathcal{B}}=$ $\left\{\underline{m}=\left[m_{\mathcal{A}}, m_{\mathcal{B}}\right]: m_{\mathcal{A}} \in \mathcal{M}_{\mathcal{A}}, m_{\mathcal{B}} \in \mathcal{M}_{\mathcal{B}}\right\}$. Given $\underline{M}(t)=\underline{m}=\left[m_{\mathcal{A}}, m_{\mathcal{B}}\right]$, we assume that the past defaults in $\mathcal{A}$ and $\mathcal{B}$ would generate the default intensities of individual active corporations in $\mathcal{A}$ and $\mathcal{B}$ in such a way that those intensities are proportional to the number of defaults occurred by time $t$. More specifically, the default intensity of active corporations in $\mathcal{A}$ reflecting the second factor is given by

$$
\rho_{\mathcal{A} \rightarrow \mathcal{A}} \times\left(M_{\mathcal{A}}-m_{\mathcal{A}}\right)+\rho_{\mathcal{B} \rightarrow \mathcal{A}} \times\left(M_{\mathcal{B}}-m_{\mathcal{B}}\right) .
$$

Similarly, this default intensity for active corporations in $\mathcal{B}$ is given by

$$
\rho_{\mathcal{A} \rightarrow \mathcal{B}} \times\left(M_{\mathcal{A}}-m_{\mathcal{A}}\right)+\rho_{\mathcal{B} \rightarrow \mathcal{B}} \times\left(M_{\mathcal{B}}-m_{\mathcal{B}}\right) .
$$

We note that the past defaults in $\mathcal{A}$ would affect an active corporation in $\mathcal{A}$ and that in $\mathcal{B}$ differently where the default intensity for the former is given by $\rho_{\mathcal{A} \rightarrow \mathcal{A}} \times\left(M_{\mathcal{A}}-m_{\mathcal{A}}\right)$ and that for the latter is 
described by $\rho_{\mathcal{A} \rightarrow \mathcal{B}} \times\left(M_{\mathcal{A}}-m_{\mathcal{A}}\right)$. The default intensities $\rho_{\mathcal{B} \rightarrow \mathcal{A}} \times\left(M_{\mathcal{B}}-m_{\mathcal{B}}\right)$ and $\rho_{\mathcal{B} \rightarrow \mathcal{B}} \times\left(M_{\mathcal{B}}-m_{\mathcal{B}}\right)$ can be interpreted in a similar manner.

In order to develop the integrated model, of interest is the trivariate stochastic process $[J(t), \underline{M}(t)]$ defined on $\mathcal{J} \times \mathcal{M}$. Given $[i, \underline{m}]$ with $\underline{m}=\left[m_{\mathcal{A}}, m_{\mathcal{B}}\right]$, let $\eta_{\mathcal{A}}(i, \underline{m})$ and $\eta_{\mathcal{B}}(i, \underline{m})$ be the overall default intensities of an active corporation in $\mathcal{A}$ and an active corporation in $\mathcal{B}$ respectively. From Definition 2.1, (2.1) and (2.2), one then sees that

$$
\begin{aligned}
\eta_{\mathcal{A}}(i, \underline{m}) & =\xi_{\mathcal{A}}(i)+\rho_{\mathcal{A} \rightarrow \mathcal{A}} \times\left(M_{\mathcal{A}}-m_{\mathcal{A}}\right)+\rho_{\mathcal{B} \rightarrow \mathcal{A}} \times\left(M_{\mathcal{B}}-m_{\mathcal{B}}\right), \\
\eta_{\mathcal{B}}(i, \underline{m}) & =\xi_{\mathcal{B}}(i)+\rho_{\mathcal{A} \rightarrow \mathcal{B}} \times\left(M_{\mathcal{A}}-m_{\mathcal{A}}\right)+\rho_{\mathcal{B} \rightarrow \mathcal{B}} \times\left(M_{\mathcal{B}}-m_{\mathcal{B}}\right) .
\end{aligned}
$$

Given $[i, \underline{m}]$, let the integrated industrial default intensities for $\mathcal{A}$ and $\mathcal{B}$ be denoted by $\lambda_{\mathcal{A}}(i, \underline{m})$ and $\lambda_{\mathcal{B}}(i, \underline{m})$ respectively. Since there are $m_{\mathcal{A}}$ and $m_{\mathcal{B}}$ active corporations in $\mathcal{A}$ and $\mathcal{B}$, one has

$$
\begin{aligned}
\lambda_{\mathcal{A}}(i, \underline{m}) & =m_{\mathcal{A}} \times \eta_{\mathcal{A}}(i, \underline{m}), \\
\lambda_{\mathcal{B}}(i, \underline{m}) & =m_{\mathcal{B}} \times \eta_{\mathcal{B}}(i, \underline{m}) .
\end{aligned}
$$

As before, we summarize the above definitions which would play a key role in our analysis.

\section{Definition 2.2}

(a) $\eta_{\mathcal{A}}(i, \underline{m})\left[\eta_{\mathcal{B}}(i, \underline{m})\right]$ : the default intensity of an active corporation in $\mathcal{A}[\mathcal{B}]$ triggered by the macro economic factor as well as the past defaults in $\mathcal{A}$ and $\mathcal{B}$ when $J(t)=i$ and $\underline{M}(t)=\underline{m}$

(b) $\lambda_{\mathcal{A}}(i, \underline{m})\left[\lambda_{\mathcal{B}}(i, \underline{m})\right]$ : the integrated default intensity of the industrial sector $\mathcal{A}[\mathcal{B}]$ triggered by the macro economic factor as well as the past defaults in $\mathcal{A}$ and $\mathcal{B}$ when $J(t)=i$ and $\underline{M}(t)=\underline{m}$

Of interest is the time dependent dynamic behavior of the trivariate stochastic process $[J(t), \underline{M}(t)]$, which would enable one to assess the joint distribution of the cumulative losses due to the defaults occurred in $\mathcal{A}$ and in $\mathcal{B}$ by time $t$. Given a CDO tranche $\left[K_{a}, K_{d}\right]$, these time dependent distributions then provide a computational vehicle to evaluate the price of the CDO tranche over a time period $[0, T]$ subject to the condition that the price assures the no-arbitrage condition of the CDO market. It then becomes possible to study how the underlying parameter values, representing interactions between the macro economic condition, the industrial sector $\mathcal{A}$ and the industrial sector $\mathcal{B}$, would affect the CDO prices.

\section{Fundamental Absorbing Trivariate Process and Its First Passage Time Structure}

In this section, we analyze the dynamic behavior of the trivariate process $[J(t), \underline{M}(t)]=\left[J(t),\left(M_{\mathcal{A}}(t), M_{\mathcal{B}}(t)\right)\right]$ in detail. As we will see soon, the trivariate process can be formulated as a sophisticated bivariate MMPP(Markov Modulated Poisson Process) with respect to two industrial sectors $\mathcal{A}$ and $\mathcal{B}$, which is both temporally and spatially inhomogeneous. Based on the uniformization procedure of Keilson [7] combined with the dynamic first passage time analysis, the computational algorithms will be developed for evaluating the joint distribution of the cumulative losses due to the defaults occurred in $\mathcal{A}$ and in $\mathcal{B}$ by time $t$.

In order to capture the dynamic behavior of the bivariate MMPP, we introduce a fundamental absorbing trivariate process $\left[J(t), \underline{M}_{m}(t)\right]$ given $\underline{m}=\left(m_{\mathcal{A}}, m_{\mathcal{B}}\right)>\underline{0}$, where the corresponding state space is given by

$(3.1) \mathcal{S}_{\underline{m}} \stackrel{\text { def }}{=} \mathcal{J} \times \mathcal{M}_{\underline{m}}=\left\{(i, \underline{n}): i \in \mathcal{J}, \underline{n}=\left(m_{\mathcal{A}}, m_{\mathcal{B}}\right),\left(m_{\mathcal{A}}-1, m_{\mathcal{B}}\right)\right.$ or $\left.\left(m_{\mathcal{A}}, m_{\mathcal{B}}-1\right)\right\}$.

The fundamental absorbing trivariate process is constructed in such a way that its stochastic behavior is identical to that of $[J(t), \underline{M}(t)]$ if $\underline{M}_{\underline{m}}(t)=\underline{m}$, while all other states are made absorbing. 
More specifically, the transition structure of $\left[J(t), \underline{M}_{\underline{m}}(t)\right]$ is depicted in Figure 3.1 with corresponding hazard rates listed in Table 3.1. If $m_{\mathcal{A}}=0$ or $m_{\mathcal{B}}=0$, or both, states with negative index in the above definitions would be ignored. With this modification, the arguments to follow would be valid for $\underline{m} \geq \underline{0}$, which we assume throughout the rest of the paper.

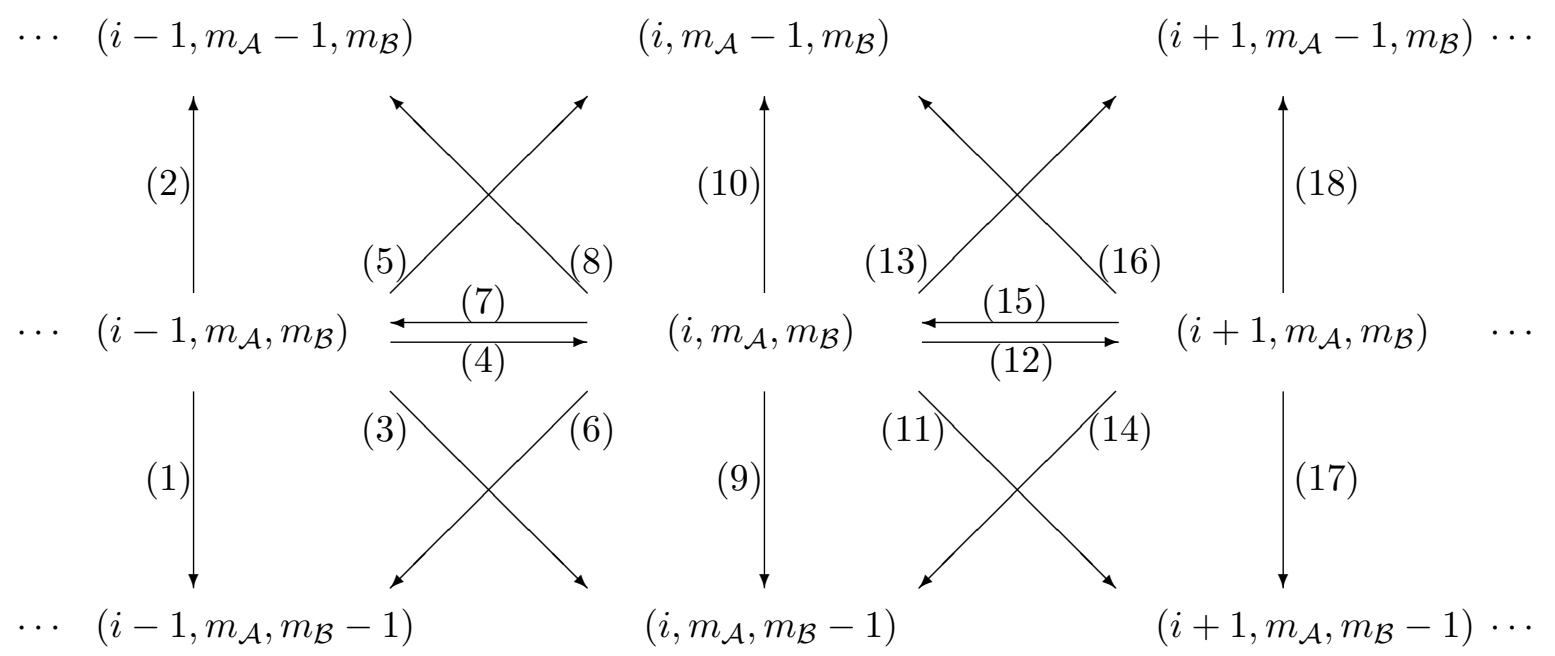

Figure 3.1: Transition Structure of $[J(t), \underline{M}(t)]$
(1): $\lambda_{\mathcal{B}}(i-1, \underline{m})$
(5): $\nu_{i-1}^{+} \Theta_{i-1, i}^{\mathcal{A}}$
(9): $\lambda_{\mathcal{B}}(i, \underline{m})$
(13): $\nu_{i}^{+} \Theta_{i, i+1}^{\mathcal{A}}$
$(17): \lambda_{\mathcal{B}}(i+1, \underline{m})$
(2): $\lambda_{\mathcal{A}}(i-1, \underline{m})$
(6): $\nu_{i}^{-} \Theta_{i, i-1}^{\mathcal{B}}$
(10): $\lambda_{\mathcal{A}}(i, \underline{m})$
(14): $\nu_{i+1}^{-} \Theta_{i+1, i}^{\mathcal{B}}$
(18): $\lambda_{\mathcal{A}}(i+1, \underline{m})$
(3): $\nu_{i-1}^{+} \Theta_{i-1, i}^{\mathcal{B}}$
(7): $\nu_{i}^{-} \Theta_{i, i-1}$
(11): $\nu_{i}^{+} \Theta_{i, i+1}^{\mathcal{B}}$
(15): $\nu_{i+1}^{-} \Theta_{i+1, i}$
(4): $\nu_{i-1}^{+} \Theta_{i-1, i}$
(8): $\nu_{i}^{-} \Theta_{i, i-1}^{\mathcal{A}}$
(12): $\nu_{i}^{+} \Theta_{i, i+1}$
(16): $\nu_{i+1}^{-} \Theta_{i+1, i}^{\mathcal{A}}$

Table 3.1: Associated Hazard Rates

In order to define $\left[J(t), \underline{M}_{m}(t)\right]$ in terms of hazard rate matrices, we decompose the state space $\mathcal{S}_{\underline{m}}$ in (3.1) into three mutually exclusive and exhaustive subspaces in the following manner.

$$
\begin{array}{lll}
\mathcal{S}_{\underline{m}}^{0} & \stackrel{\text { def }}{=} & \left\{(i, \underline{n}): i \in \mathcal{J}, \underline{n}=\left(m_{\mathcal{A}}, m_{\mathcal{B}}\right)\right\} \\
\mathcal{S}_{\underline{m}}^{\mathcal{A}} & \stackrel{\text { def }}{=} & \left\{(i, \underline{n}): i \in \mathcal{J}, \underline{n}=\left(m_{\mathcal{A}}-1, m_{\mathcal{B}}\right)\right\} \\
\mathcal{S}_{\underline{m}}^{\mathcal{B}} & \stackrel{\text { def }}{=} & \left\{(i, \underline{n}): i \in \mathcal{J}, \underline{n}=\left(m_{\mathcal{A}}, m_{\mathcal{B}}-1\right)\right\}
\end{array}
$$

It can be seen that $\mathcal{S}_{\underline{m}}^{\mathcal{A}}$ corresponds to the set of states located in the first row of Figure 3.1. Similarly, those states in the second row and those in the third row of Figure 3.1 constitute $\mathcal{S}_{\underline{m}}^{0}$ and $\mathcal{S}_{m}^{\mathcal{B}}$ respectively.

With this definition, let $\underline{\underline{\nu}}$ be the hazard rate matrix governing the transitions of $\left[J(t), \underline{M}_{m}(t)\right]$ within $\mathcal{S}_{\underline{m}}^{0}$. Based on the discussions in Section2, one sees that

$$
\underline{\nu} \stackrel{\text { def }}{=}\left[\begin{array}{cccccc}
0 & \nu_{0}^{+} \Theta_{0,1} & & & & \\
\nu_{1}^{-} \Theta_{1,0} & 0 & \nu_{1}^{+} \Theta_{1,2} & & & \\
& \ddots & \ddots & \ddots & & \\
& & \nu_{i}^{-} \Theta_{i, i-1} & 0 & \nu_{i}^{+} \Theta_{i, i+1} & \\
& & & \ddots & \ddots & \\
& & & & \nu_{J}^{-} \Theta_{J, J-1} & 0
\end{array}\right] .
$$

It is worth noting that $\underline{\underline{\nu}}$ is independent of $\underline{m}$ as indicated by its notation, provided that $0<m_{\mathcal{A}}<$ $M_{\mathcal{A}}$ and $0<m_{\mathcal{B}}<M_{\mathcal{B}}$. In order to capture the transitions of $\left[J(t), \underline{M}_{m}(t)\right]$ from $\mathcal{S}_{m}^{0}$ to $\mathcal{S}_{\underline{m}}^{\mathcal{A}}$ and 
those from $\mathcal{S}_{\underline{m}}^{0}$ to $\mathcal{S}_{\underline{m}}^{\mathcal{B}}$, we define the two matrices $\underline{\underline{\Lambda}}_{\mathcal{A}}(\underline{m})$ and $\underline{\underline{\Lambda}}_{\mathcal{B}}(\underline{m})$ by

$$
\underline{\Lambda}_{\mathcal{A}}(\underline{m}) \stackrel{\text { def }}{=}\left[\begin{array}{cccccc}
\lambda_{\mathcal{A}}(0, \underline{m}) & \nu_{0}^{+} \Theta_{0,1}^{\mathcal{A}} & & & & \\
\nu_{1}^{-} \Theta_{1,0}^{\mathcal{A}} & \lambda_{\mathcal{A}}(1, \underline{m}) & \nu_{1}^{+} \Theta_{1,2}^{\mathcal{A}} & & & \\
& \ddots & \ddots & \ddots & & \\
& & \nu_{i}^{-} \Theta_{i, i-1}^{\mathcal{A}} & \lambda_{\mathcal{A}}(i, \underline{m}) & \nu_{i}^{+} \Theta_{i, i+1}^{\mathcal{A}} & \\
& & & \ddots & \ddots & \\
& & & & \nu_{J}^{-} \Theta_{J, J-1}^{\mathcal{A}} & \lambda_{\mathcal{A}}(J, \underline{m})
\end{array}\right],
$$

and

$$
\underline{\underline{\Lambda}}_{\mathcal{B}}(\underline{m}) \stackrel{\text { def }}{=}\left[\begin{array}{cccccc}
\lambda_{\mathcal{B}}(0, \underline{m}) & \nu_{0}^{+} \Theta_{0,1}^{\mathcal{B}} & & & & \\
\nu_{1}^{-} \Theta_{1,0}^{\mathcal{B}} & \lambda_{\mathcal{B}}(1, \underline{m}) & \nu_{1}^{+} \Theta_{1,2}^{\mathcal{B}} & & & \\
& \ddots & \ddots & \ddots & & \\
& & \nu_{i}^{-} \Theta_{i, i-1}^{\mathcal{B}} & \lambda_{\mathcal{B}}(i, \underline{m}) & \nu_{i}^{+} \Theta_{i, i+1}^{\mathcal{B}} & \\
& & & \ddots & \ddots & \\
& & & & \nu_{J}^{-} \Theta_{J, J-1}^{\mathcal{\mathcal { B }}} & \lambda_{\mathcal{B}}(J, \underline{m})
\end{array}\right] .
$$

The entire hazard rate matrix governing $\left[J(t), \underline{M}_{\underline{m}}(t)\right]$, denoted by $\underline{\underline{\mathcal{V}}}(\underline{m})$, can then be written as

$$
\begin{aligned}
& \mathcal{S}_{\underline{m}}^{0} \quad \mathcal{S}_{\underline{m}}^{\mathcal{A}} \quad \mathcal{S}_{\underline{m}}^{\mathcal{B}} \\
& \underline{\underline{\mathcal{V}}}(\underline{m}) \stackrel{\text { def }}{=} \quad \begin{array}{c}
\mathcal{S}_{\underline{m}}^{0} \\
\mathcal{S}_{m}^{\mathcal{A}} \\
\\
\mathcal{S}_{\underline{m}}^{\mathcal{B}}
\end{array}\left[\begin{array}{ccc}
\underline{\underline{\nu}} & \underline{\Lambda}_{\mathcal{A}}(\underline{\underline{m}}) & \underline{\underline{\Lambda}}_{\mathcal{B}}(\underline{m}) \\
\underline{\underline{0}} & \underline{\underline{0}} & \underline{\underline{0}} \\
\underline{\underline{0}} & \underline{\underline{0}} & \underline{\underline{0}}
\end{array}\right] .
\end{aligned}
$$

We are now in a position to develop computational procedures for evaluating the dynamic transition probabilities of $\left[J(t), \underline{M}_{m}(t)\right]$ based on the uniformization procedure of Keilson[7]. More specifically, let

$$
P_{(i, \underline{n})\left(i^{\prime}, \underline{n}^{\prime}\right)}(\underline{m}, t)=\mathbb{P}\left[J(t)=i^{\prime}, M_{\underline{m}}(t)=\underline{n}^{\prime} \mid J(0)=i, M_{\underline{m}}(0)=\underline{n}\right]
$$

and define the transition probability matrix

$$
\underline{\underline{P}}(\underline{m}, t)=\left[P_{(i, \underline{n})\left(i^{\prime}, \underline{n}^{\prime}\right)}(\underline{m}, t)\right]_{(i, \underline{n}),\left(i^{\prime}, \underline{n}^{\prime}\right) \in \mathcal{S}_{\underline{m}}},
$$

where the state space $\mathcal{S}_{\underline{m}}$ is arranged in the order of $\mathcal{S}_{\underline{m}}^{0}, \mathcal{S}_{\underline{m}}^{\mathcal{A}}$ and $\mathcal{S}_{\underline{m}}^{\mathcal{B}}$ as for $\underline{\underline{\mathcal{V}}}(\underline{m})$ given in (3.6). The corresponding Laplace transform is defined by

$$
\underline{\underline{\pi}}(\underline{m}, s)=\int_{0}^{t} e^{-s t} \underline{\underline{P}}(\underline{m}, t) d t
$$

For notational convenience, given a matrix $\underline{\underline{\zeta}}=\left[\zeta_{i j}\right]_{i, j \in \mathcal{N}}$, we define the $i$-th row sum by $\zeta_{i}$ and the associated diagonal matrix by $\underline{\underline{\zeta}}_{D}$, i.e.

$$
\underline{\underline{\zeta}}_{D}=\operatorname{diag}\left\{\zeta_{i}\right\} ; \zeta_{i}=\sum_{j \in \mathcal{N}} \zeta_{i j} .
$$

With this definition, for $\underline{\underline{\mathcal{V}}}(\underline{m})$, one has

$$
\underline{\underline{\mathcal{V}}}_{D}(\underline{m})=\left[\begin{array}{ccc}
\underline{\underline{\nu}}_{D}+\underline{\underline{\Lambda}}_{\mathcal{A}: D}(\underline{\underline{m}})+\underline{\underline{\Lambda}}_{\mathcal{B}: D}(\underline{m}) & \underline{\underline{0}} & \underline{\underline{0}} \\
\underline{\underline{0}} & \underline{\underline{0}} & \underline{\underline{0}} \\
\underline{\underline{0}} & \underline{\underline{0}} & \underline{\underline{0}}
\end{array}\right] .
$$


The infinitesimal generator $\underline{\underline{\mathcal{Q}}}(\underline{m})$ of $\left[J(t), \underline{M}_{\underline{m}}(t)\right]$ can then be given by

$$
\underline{\underline{\mathcal{Q}}}(\underline{m})=-\underline{\underline{\mathcal{V}}}_{D}(\underline{m})+\underline{\underline{\mathcal{V}}}(\underline{m}),
$$

where $\underline{\underline{\mathcal{V}}}(\underline{m})$ is as in (3.6). Accordingly, the Kolmogorov forward equation is obtained as

$$
\frac{d}{d t} \underline{\underline{P}}(\underline{m}, t)=\underline{\underline{P}}(\underline{m}, t) \underline{\underline{\mathcal{Q}}}(\underline{m}) .
$$

By taking the Laplace transform of both sides of (3.13) with respect to $t$ and using $\underline{\underline{P}}(\underline{m}, 0)=\underline{\underline{I}}$, one sees that

$$
s \underline{\underline{\pi}}(\underline{m}, s)-\underline{\underline{I}}=\underline{\underline{\pi}}(\underline{m}, s) \underline{\underline{\mathcal{Q}}}(\underline{m}),
$$

which in turn leads to

$$
\underline{\underline{\pi}}(\underline{m}, s)=[s \underline{\underline{I}}-\underline{\underline{\mathcal{Q}}}(\underline{m})]^{-1} .
$$

In the real domain, Equation (3.15) implies that

$$
\underline{\underline{P}}(\underline{m}, t)=\exp (\underline{\underline{\mathcal{Q}}}(\underline{m}) t)=\sum_{k=0}^{\infty} \frac{\underline{\underline{Q}}(\underline{\underline{m}})^{k}}{k !} t^{k} .
$$

In order to facilitate our analysis further, we introduce the uniformization procedure of Keilson [7]. Given $\underline{m} \in \mathcal{M}$, let $\nu$ be a constant satisfying

$$
\nu \geq \max _{[i, \underline{m}] \in \mathcal{J} \times \mathcal{M}}\left\{\nu_{i}+\lambda_{\mathcal{A}}(i, \underline{m})+\lambda_{\mathcal{B}}(i, \underline{m})\right\} .
$$

The stochastic matrix $\underline{\underline{a}}_{\nu}(\underline{m})$ associated with $\nu$ is then defined as

$$
\underline{\underline{a}}_{\nu}(\underline{m}) \stackrel{\text { def }}{=} \underline{\underline{I}}-\frac{1}{\nu} \underline{\underline{\mathcal{V}}}_{D}(\underline{m})+\frac{1}{\nu} \underline{\underline{\mathcal{V}}}(\underline{m}) .
$$

One sees from (3.10) and (3.18) that $\underline{\underline{a}}_{\nu}(\underline{m})=\underline{\underline{I}}+\frac{1}{\nu} \underline{\underline{\mathcal{Q}}}(\underline{\underline{m}})$. Substitution of this equation into (3.15) then yields

$$
\underline{\underline{\pi}}(\underline{m}, s)=\frac{1}{s+\nu}\left[\underline{\underline{I}}-\frac{\nu}{s+\nu} \underline{\underline{a}}(\underline{m})\right]^{-1} .
$$

The uniformization procedure characterized by (3.17) through (3.19) has the following probabilistic interpretation. In general, the dwell time of the trivariate process $\left[J(t), \underline{M}_{\underline{m}}(t)\right]$ at state $[i, \underline{m}]$ is exponentially distributed with parameter $\nu_{i}+\lambda_{\mathcal{A}}(i, \underline{m})+\lambda_{\mathcal{B}}(i, \underline{m})$, which depends on $[i, \underline{m}]$. The next transition is then governed by the matrix $\left[\underline{\underline{\nu}}_{D}+\underline{\underline{\Lambda}}_{\mathcal{A}: D}(\underline{m})+\underline{\underline{\Lambda}}_{\mathcal{B}: D}(\underline{m})\right]^{-1}\left[\underline{\underline{\nu}}+\underline{\underline{\Lambda}}_{\mathcal{A}}(\underline{m})+\underline{\underline{\Lambda}}_{\mathcal{B}}(\underline{m})\right]$. Replacing the dwell time at state $[i, \underline{m}]$ by the exponential random variate with parameter $\nu$ in (3.17) for all $[i, \underline{m}] \in \mathcal{S}_{\underline{m}}$, the uniformization procedure states that the resulting Markov chain can be made probabilistically identical to the original Markov chain where $\underline{\underline{a}}_{\nu}(\underline{m})$ in (3.15) is employed for the probability matrix dictating the next transition.

We now prove the key theorem of this section. For notational simplicity, the relevant subspaces of the state space of $\left[J(t), M_{\underline{m}}(t)\right]$ are defined as follows.

$$
G \stackrel{\text { def }}{=} \mathcal{S}_{\underline{m}}^{0} ; A \stackrel{\text { def }}{=} \mathcal{S}_{\underline{m}}^{\mathcal{A}} ; B \stackrel{\text { def }}{=} \mathcal{S}_{\underline{m}}^{\mathcal{B}} .
$$

Let $\underline{\underline{a}}$ be a matrix corresponding to $\mathcal{S}_{\underline{m}} \times \mathcal{S}_{\underline{m}}$. A submatrix of $\underline{\underline{a}}$ restricted to $G \times G$ is denoted by

$$
\underline{\underline{a}}_{G G}=\left[a_{[i, \underline{m}][j, \underline{m}]}\right]_{[i, \underline{m}] \in G,[j, \underline{m}] \in G} .
$$

Since the cardinality of $G$ is equal to the cardinality of $\mathcal{J}$ from (3.2), for notational simplicity, we write $a_{[i, \underline{m}][j, \underline{m}]}=a_{i j}$. Submatrices $\underline{\underline{a}}_{G A}$ and $\underline{\underline{a}}_{G B}$ are defined similarly. 
Theorem 3.1 Let $\underline{\underline{\pi}}(\underline{m}, s)$ be as in (3.9). With $G, A$ and $B$ as defined in (3.20), the following statements hold.

$$
\begin{array}{ll}
\text { a) } & \underline{\underline{\pi}}_{G G}(\underline{m}, s)=\frac{1}{s+\nu}\left[\sum_{k=0}^{\infty}\left(\frac{\nu}{s+\nu}\right)^{k} \underline{\underline{a}}_{\nu: G G}(\underline{m})^{k}\right] \\
\text { b) } & \underline{\underline{\pi}}_{G A}(\underline{\underline{m}}, s)=\frac{1}{s+\nu}\left[\sum_{k=0}^{\infty}\left(\frac{\nu}{s+\nu}\right)^{k}\left(\sum_{j=0}^{k-1} \underline{\underline{a}}_{\nu: G G}\left(\underline{m}^{j} \underline{\underline{a}}_{\nu: G A}(\underline{m})\right)\right]\right. \\
\text { c) } & \underline{\underline{\pi}}_{G B}(\underline{m}, s)=\frac{1}{s+\nu}\left[\sum_{k=0}^{\infty}\left(\frac{\nu}{s+\nu}\right)^{k}\left(\sum_{j=0}^{k-1} \underline{\underline{a}}_{\nu: G G}(\underline{m})^{j} \underline{\underline{a}}_{\nu: G B}(\underline{m})\right)\right]
\end{array}
$$

\section{Proof}

From $(3.6),(3.11)$ and $(3.18)$, it can be seen that $\underline{\underline{a}}_{\nu}(\underline{m})$ takes the form

$$
\underline{\underline{a}}_{\nu}(\underline{m})=\left(\begin{array}{ccc}
\underline{\underline{a}}_{\nu: G G}(\underline{\underline{m}}) & \underline{\underline{a}}_{\nu: G A}(\underline{\underline{m}}) & \underline{\underline{a}}_{\nu: G B}(\underline{\underline{m}}) \\
\underline{\overline{0}} & \underline{\overline{0}} & \underline{\overline{0}} \\
\underline{\underline{0}} & \underline{\underline{0}} & \underline{\underline{0}}
\end{array}\right) .
$$

By induction, after a little algebra, it then follows that

$$
\underline{\underline{a}}_{\nu}(\underline{m})^{k}=\left(\begin{array}{ccc}
\underline{\underline{a}}_{\nu: G G}(\underline{\underline{m}})^{k} & \underline{\underline{a}}_{\nu: G G}(\underline{\underline{m}})^{k-1} \underline{\underline{a}}_{\nu: G A}(\underline{\underline{m}}) & \underline{\underline{a}}_{\nu: G G}(\underline{\underline{m}})^{k-1} \underline{\underline{a}}_{\nu: G B}(\underline{\underline{m}}) \\
\underline{\underline{\underline{0}}} & \underline{\underline{0}} & \underline{\underline{0}}
\end{array}\right) .
$$

The theorem now follows from (3.19).

As we will see, Theorem 3.1 enables one to evaluate the distribution of the first passage time of $\left[J(t), \underline{M}_{\underline{m}}(t)\right]$ from $[i, \underline{n}]$ to $\left[i^{\prime}, \underline{n}^{\prime}\right]$, thereby providing a computational vehicle for evaluating the CDO prices of our model. More specifically, let $\mathcal{T}_{[i, \underline{n}] \rightarrow A \cup B}$ be the first passage time of $\left[J(t), \underline{M}_{\underline{m}}(t)\right]$ from $[i, \underline{n}]$ to $A \cup B$, i.e.

$$
\mathcal{T}_{[i, \underline{n}] \rightarrow A \cup B}=\inf \left\{t:\left[J(t), \underline{M}_{\underline{m}}(t)\right] \in A \cup B \mid\left[J(0), \underline{M}_{\underline{m}}(0)\right]=[i, \underline{n}]\right\} .
$$

We introduce the following indicator

$$
I\left(\mathcal{T}_{[i, \underline{n}] \rightarrow A \cup B}\right)=\left\{\begin{array}{lll}
A & \text { if } & \underline{M}_{m} \\
B & \text { if } \underline{M}_{\underline{m}}\left(\mathcal{T}_{[i, \underline{n}] \rightarrow A \cup B}\right) \in A \\
\left.\mathcal{T}_{[i, \underline{n}] \rightarrow A \cup B}\right) \in B
\end{array}\right.
$$

and define the joint distribution matrices of $\mathcal{T}_{[i, \underline{n}] \rightarrow A \cup B}$ and $I\left(\mathcal{T}_{[i, \underline{n}] \rightarrow A \cup B}\right)$ as

$$
\begin{aligned}
\underline{S}_{A}(\underline{m}, t) & =\left[S_{A: i j}(\underline{m}, t)\right] \\
S_{A: i j}(\underline{m}, t) & =\mathbb{P}\left\{\mathcal{T}_{[i, \underline{n}] \rightarrow A \cup B} \leq t, I\left(\mathcal{T}_{[i, \underline{n}] \rightarrow A \cup B}\right)=A, J(t)=j \mid \underline{M}_{\underline{m}}(0)=\underline{n}, J(0)=i\right\}
\end{aligned}
$$

and

$$
\begin{aligned}
\underline{S}_{B}(\underline{m}, t) & =\left[S_{B: i j}(\underline{m}, t)\right] \\
S_{B: i j}(\underline{m}, t) & =\mathbb{P}\left\{\mathcal{T}_{[i, \underline{n}] \rightarrow A \cup B} \leq t, I\left(\mathcal{T}_{[i, \underline{n}] \rightarrow A \cup B}\right)=B, J(t)=j \mid \underline{M}_{\underline{m}}(0)=\underline{n}, J(0)=i\right\} .
\end{aligned}
$$

The corresponding Laplace transform matrices are denoted by

$$
\underline{\underline{\sigma}}_{A}(\underline{m}, s) \stackrel{\text { def }}{=}\left[\sigma_{A: i j}(\underline{m}, s)\right] ; \sigma_{A: i j}(\underline{m}, s)=\int_{0}^{\infty} e^{-s t} d S_{A: i j}(\underline{m}, t)
$$


and

$$
\underline{\underline{\sigma}}_{B}(\underline{m}, s) \stackrel{\text { def }}{=}\left[\sigma_{B: i j}(\underline{m}, s)\right] ; \sigma_{B: i j}(\underline{m}, s)=\int_{0}^{\infty} e^{-s t} d S_{B: i j}(\underline{m}, t) .
$$

The next theorem then holds true.

\section{Theorem 3.2}

$$
\begin{aligned}
\text { a) } \underline{\underline{\sigma}}_{A}(\underline{\underline{m}}, s) & =\sum_{k=0}^{\infty}\left(\frac{\nu}{s+\nu}\right)^{k+1} \underline{\underline{a}}_{\nu: G G}(\underline{m})^{k} \underline{\underline{a}}_{\nu: G A}(\underline{\underline{m}}) ; \\
\underline{\underline{\sigma}}_{B}(\underline{\underline{m}}, s) & =\sum_{k=0}^{\infty}\left(\frac{\nu}{s+\nu}\right)^{k+1} \underline{\underline{a}}_{\nu: G G}(\underline{\underline{m}})^{k} \underline{\underline{a}}_{\nu: G B}(\underline{m}), \\
\text { b) } \quad \underline{\underline{s}}_{A}(\underline{\underline{m}}, \tau) & =\sum_{k=0}^{\infty} e^{-\nu \tau} \frac{(\nu \tau)^{k}}{k !} \underline{\underline{a}}_{\nu: G G}(\underline{\underline{m}})^{k} \underline{\underline{a}}_{\nu: G A}(\underline{m}) ; \\
\underline{\underline{s}}_{B}(\underline{\underline{m}}, \tau) & =\sum_{k=0}^{\infty} e^{-\nu \tau} \frac{(\nu \tau)^{k}}{k !} \underline{\underline{a}}_{\nu: G G}(\underline{\underline{m}})^{k} \underline{\underline{a}}_{\nu: G B}(\underline{\underline{m}}) .
\end{aligned}
$$

\section{Proof}

From the probabilistic interpretation of the uniformization procedure, one sees that

$$
\begin{aligned}
& \sigma_{A: i j}(\underline{m}, s)=\frac{\nu}{s+\nu}\left[\delta_{\{i=j\}} a_{\nu:\left[i, m_{\mathcal{A}}, m_{\mathcal{B}}\right]\left[j, m_{\mathcal{A}}-1, m_{\mathcal{B}}\right]}(\underline{m})\right. \\
&\left.+\sum_{r \in \mathcal{J}} a_{\nu:\left[i, m_{\mathcal{A}}, m_{\mathcal{B}}\right]\left[r, m_{\mathcal{A}}, m_{\mathcal{B}}\right]}(\underline{m}) \sigma_{A: r j}(\underline{m}, s)\right],
\end{aligned}
$$

where $\delta_{\{\text {Statement }\}}=1$ if Statement holds true and $\delta_{\{\text {Statement }\}}=0$ otherwise. Equation (3.27) can be explained in the following manner. Starting from $[i, \underline{m}]=\left[J(0), \underline{M}_{m}(0)\right]$, the left hand side is the Laplace transform of the joint probability of the time until absorption and the event that this absorption occurs in $A$ with entry into $A$ at $\left[j, m_{\mathcal{A}}-1, m_{\mathcal{B}}\right]$. In order to see the probabilistic interpretation of the right hand side, we first note from the uniformization procedure that the dwell time in state $[i, \underline{m}]$ can be considered to have the exponential distribution with the Laplace transform $\nu /(s+\nu)$. The absorbing set $A$ can be reached directly from $[i, \underline{m}]$ only through $\left[i, m_{\mathcal{A}}-1, m_{\mathcal{B}}\right]$. Accordingly, the first transition results in absorption with probability $a_{\nu:\left[i, m_{\mathcal{A}}, m_{\mathcal{B}}\right]\left[i, m_{\mathcal{A}}-1, m_{\mathcal{B}}\right]}(\underline{m})$. Otherwise, the next state to be visited would be $[r, \underline{m}]$ with probability $a_{\nu:[i, \underline{m}][r, \underline{m}]}(\underline{m})$. In this case, the state $\left[j, m_{\mathcal{A}}-1, m_{\mathcal{B}}\right]$ should be reached anew from $[r, \underline{m}]$, and the Laplace transform $\sigma_{A: r j}(\underline{m}, s)$ should be multiplied. In matrix form, Equation (3.27) can be rewritten as

$$
\underline{\underline{\sigma}}_{A}(\underline{\underline{m}}, s)=\frac{\nu}{s+\nu}\left[\underline{\underline{a}}_{\nu: G A}(\underline{\underline{m}})+\underline{\underline{a}}_{\nu: G G}(\underline{m}) \underline{\underline{\sigma}}_{A}(\underline{\underline{m}}, s)\right],
$$

which can be solved for $\underline{\underline{\sigma}}_{A}(\underline{m}, s)$ as

$$
\underline{\underline{\sigma}}_{A}(\underline{m}, s)=\frac{\nu}{s+\nu}\left[\underline{\underline{I}}-\frac{\nu}{s+\nu} \underline{\underline{a}}_{\nu: G G}(\underline{m})\right]^{-1} \underline{a}_{\nu: G A}(\underline{m}) .
$$

Similarly, $\underline{\underline{\sigma}}_{B}(\underline{m}, s)$ can be obtained as

$$
\underline{\underline{\sigma}}_{B}(\underline{m}, s)=\frac{\nu}{s+\nu}\left[\underline{\underline{I}}-\frac{\nu}{s+\nu} \underline{\underline{a}}_{\nu: G G}(\underline{m})\right]^{-1} \underline{\underline{a}}_{\nu: G B}(\underline{m}) .
$$

Part a) then follows by expanding (3.29) and (3.30) into a geometric series. The explicit inversion of part a) into the real domain yields part b), completing the proof. 


\section{Time Dependent Joint Probability of $[J(t), \underline{M}(t)]$}

In this section, we evaluate the time dependent joint probability of $[J(t), \underline{M}(t)]$ in terms of the first passage times of the fundamental absorption processes analyzed in Section 2. This expression facilitates the necessary computations for pricing the underlying CDO subsequently as we will see. More formally, for $i, j \in \mathcal{J}$ and $\underline{m} \in \mathcal{M}$, let $b_{i j}(\underline{m}, t \mid \underline{M})$ be defined by

$$
b_{i j}(\underline{m}, t \mid \underline{M})=\mathbb{P}\{J(t)=j, \underline{M}(t)=\underline{m} \mid J(0)=i, \underline{M}(0)=\underline{M}\} .
$$

The Laplace transform with respect to $t$ is denoted by

$$
\beta_{i j}(\underline{m}, s \mid \underline{M})=\int_{0}^{\infty} e^{-s t} b_{i j}(\underline{m}, t \mid \underline{M}) d t .
$$

The corresponding matrix function and the matrix Laplace transform are written as

$$
\underline{\underline{b}}(\underline{m}, t \mid \underline{M})=\left[b_{i j}(\underline{m}, t \mid \underline{M})\right] ; \underline{\underline{\beta}}(\underline{m}, s \mid \underline{M})=\left[\underline{\underline{\beta}}_{i j}(\underline{m}, s \mid \underline{M})\right] \text {. }
$$

In what follows, we express $\underline{\beta}(\underline{m}, s \mid \underline{M})$ in a recursive form with respect to $\underline{m}$ in terms of $\underline{\underline{\pi}}(\underline{m}, s)$, $\underline{\underline{\sigma}}_{A}(\underline{m}, s)$ and $\underline{\underline{\sigma}}_{B}(\underline{m}, s)$ given in (3.19), (3.25) and (3.26) respectively.

We recall from Section 2 that, given $[J(t), \underline{M}(t)]=[i, \underline{m}]$, this trivariate process is probabilistically identical to $\left[J(t), \underline{M}_{m}\right]$ until the latter falls into absorption. Hence, the next transition of $[J(t), \underline{M}(t)]$ from state $[i, \underline{m}]$ is governed by the transition probability matrix $\underline{\underline{a}}_{\nu}(\underline{m})$ in (3.18). This transition structure is depicted in Figure 4.1.

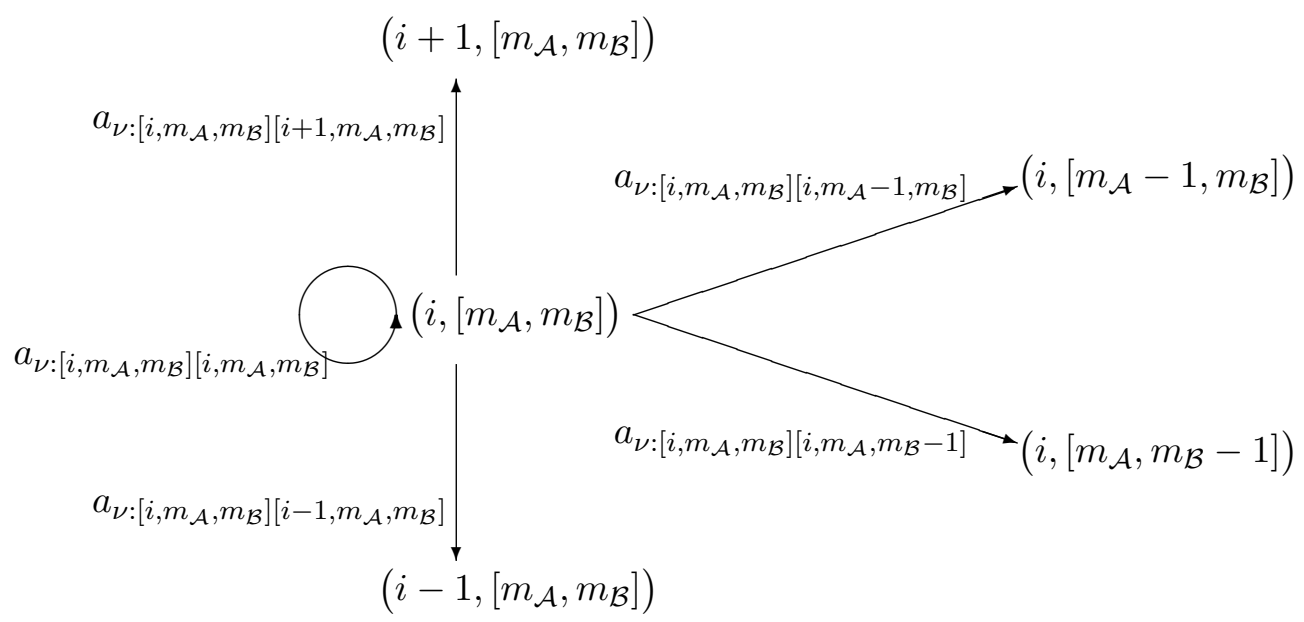

Figure 4.1: General Transition Structure of $[J(t), \underline{M}(t)]$ via Uniformization

In our model, the trivariate process $[J(t), \underline{M}(t)]$ starts with $J(0)=i \in \mathcal{J}$ and $\underline{M}(0)=\underline{M}=$ $\left[M_{\mathcal{A}}, M_{\mathcal{B}}\right]$. No default would have occurred by time $t$ if and only if $[J(t), \underline{M}(t)]$ remains within $G$ until time $t$. Accordingly, one has

$$
\underline{\underline{\beta}}\left(M_{\mathcal{A}}, M_{\mathcal{B}}, s \mid \underline{M}\right)=\underline{\underline{\pi}}_{G G}\left(M_{\mathcal{A}}, M_{\mathcal{B}}, s\right) .
$$

If there has been exactly one default in $\mathcal{A}$ by time $t$, such a default should have occurred at time $\tau<t$ and there have been no default since then until time $t$. It then follows that

$$
\underline{\underline{\beta}}\left(M_{\mathcal{A}}-1, M_{\mathcal{B}}, s \mid \underline{M}\right)=\underline{\underline{\sigma}}_{A}\left(M_{\mathcal{A}}, M_{\mathcal{B}}, s\right) \underline{\underline{\pi}}_{G G}\left(M_{\mathcal{A}}-1, M_{\mathcal{B}}, s\right) .
$$

The case of having exactly one default in $\mathcal{B}$ by time $t$ can be treated similarly and one has

$$
\underline{\underline{\beta}}\left(M_{\mathcal{A}}, M_{\mathcal{B}}-1, s \mid \underline{M}\right)=\underline{\underline{\sigma}}_{B}\left(M_{\mathcal{A}}, M_{\mathcal{B}}, s\right) \underline{\underline{\pi}}_{G G}\left(M_{\mathcal{A}}, M_{\mathcal{B}}-1, s\right) .
$$


In order to see the underlying recursive structure clearly, we continue to consider the case of having exactly two defaults by time $t$. If the two defaults occurred in $\mathcal{A}$ by time $t$, the first default in $\mathcal{A}$ should have occurred at time $\tau_{1}<t$, followed by the second default in $\mathcal{A}$ at time $\tau_{2}$ with $\tau_{1}<\tau_{2}<t$, and there should have been no default since then until time $t$. One then has

$$
\underline{\underline{\beta}}\left(M_{\mathcal{A}}-2, M_{\mathcal{B}}, s \mid \underline{M}\right)=\underline{\underline{\sigma}}_{A}\left(M_{\mathcal{A}}, M_{\mathcal{B}}, s\right) \underline{\underline{\sigma}}_{A}\left(M_{\mathcal{A}}-1, M_{\mathcal{B}}, s\right) \underline{\underline{\pi}}_{G G}\left(M_{\mathcal{A}}-2, M_{\mathcal{B}}, s\right) .
$$

Similar arguments can be employed for the case that exactly one default in $\mathcal{A}$ and exactly one default in $\mathcal{B}$ have occurred by time $t$. In this case, the two defaults should have occurred in a sequence of either $\mathcal{A}$ and $\mathcal{B}$ or $\mathcal{B}$ and $\mathcal{A}$. Consequently, we observe that

$$
\begin{aligned}
\underline{\underline{\beta}}\left(M_{\mathcal{A}}-1, M_{\mathcal{B}}-1, s \mid \underline{M}\right) & =\underline{\sigma}_{A}\left(M_{\mathcal{A}}, M_{\mathcal{B}}, s\right) \underline{\underline{\sigma}}_{B}\left(M_{\mathcal{A}}-1, M_{\mathcal{B}}, s\right) \underline{\underline{\pi}}_{G G}\left(M_{\mathcal{A}}-1, M_{\mathcal{B}}-1, s\right) \\
& +\underline{\underline{\sigma}}_{B}\left(M_{\mathcal{A}}, M_{\mathcal{B}}, s\right) \underline{\underline{\sigma}}_{A}\left(M_{\mathcal{A}}, M_{\mathcal{B}}-1, s\right) \underline{\underline{\pi}}_{G G}\left(M_{\mathcal{A}}-1, M_{\mathcal{B}}-1, s\right) .
\end{aligned}
$$

The case of having exactly two defaults in $\mathcal{B}$ can be dealt with in parallel with the case for $\mathcal{A}$, and one has

$$
\underline{\underline{\beta}}\left(M_{\mathcal{A}}, M_{\mathcal{B}}-2, s \mid \underline{M}\right)=\underline{\underline{\sigma}}_{B}\left(M_{\mathcal{A}}, M_{\mathcal{B}}, s\right) \underline{\underline{\sigma}}_{B}\left(M_{\mathcal{A}}, M_{\mathcal{B}}-1, s\right) \underline{\underline{\pi}}_{G G}\left(M_{\mathcal{A}}, M_{\mathcal{B}}-2, s\right) .
$$

In general, if $[J(t), \underline{M}(t)]=[j, \underline{m}]$, starting with $[J(0), \underline{M}(0)]=[i, \underline{M}]$, there exist multiple paths connecting $\underline{M}$ to $\underline{m}$ where each path represents a sequence of occurrences of defaults in $\mathcal{A}$ and $\mathcal{B}$. This recursive structure is depicted in Figure 4.2 for the case of $M_{\mathcal{A}}>M_{\mathcal{B}}$. The



Figure 4.2: Tree Structure

transition probability associated with each path can then be obtained by multiplying the matrices $\underline{\underline{\sigma}}_{A}(\underline{n}, s)$ and $\underline{\underline{\sigma}}_{B}\left(\underline{n}^{\prime}, s\right)$ in such a way that the sequence of the path would be reflected in the order of the matrix multiplications along with choices of $\underline{n}$ and $\underline{n}^{\prime}$, followed by the final matrix multiplication by $\underline{\underline{\pi}}_{G G}(\underline{m}, s)$. More specifically, let the matrices $\underline{\Xi}\left(m_{\mathcal{A}}, m_{\mathcal{B}}, s\right)$ be defined recursively for $m_{\mathcal{A}}=M_{\mathcal{A}}-1, M_{\mathcal{A}}-2, \cdots, 0$ and $m_{\mathcal{B}}=M_{\mathcal{B}}-1, M_{\mathcal{B}}-2, \cdots, 0$ as

$$
\begin{aligned}
& \Xi\left(m_{\mathcal{A}}, M_{\mathcal{B}}, s\right)=\Xi\left(m_{\mathcal{A}}+1, M_{\mathcal{B}}, s\right) \underline{\underline{\sigma}}_{A}\left(m_{\mathcal{A}}+1, M_{\mathcal{B}}, s\right) \text {, } \\
& \underline{\Xi}\left(m_{\mathcal{A}}, m_{\mathcal{B}}, s\right)=\underline{\Xi}\left(m_{\mathcal{A}}+1, m_{\mathcal{B}}, s\right) \underline{\underline{\sigma}}_{A}\left(m_{\mathcal{A}}+1, m_{\mathcal{B}}, s\right) \\
& +\underline{\Xi}\left(m_{\mathcal{A}}, m_{\mathcal{B}}+1, s\right) \underline{\underline{\sigma}}_{B}\left(m_{\mathcal{A}}, m_{\mathcal{B}}+1, s\right) \text {, }
\end{aligned}
$$

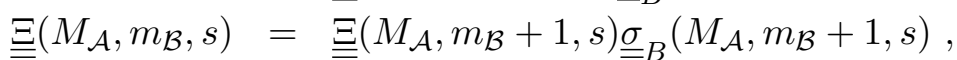

starting with

$$
\Xi\left(M_{\mathcal{A}}, M_{\mathcal{B}}, s\right)=\underline{\underline{I}} .
$$

Then the following theorem holds true. 
Theorem 4.1 For $m_{\mathcal{A}}=M_{\mathcal{A}}-1, M_{\mathcal{A}}-2, \cdots, 0$ and $m_{\mathcal{B}}=M_{\mathcal{B}}-1, M_{\mathcal{B}}-2, \cdots, 0$, let $\Xi\left(m_{\mathcal{A}}, m_{\mathcal{B}}, s\right)$ be as defined in (4.4) and (4.5). One then has

$$
\underline{\underline{\beta}}\left(m_{\mathcal{A}}, m_{\mathcal{B}}, s \mid \underline{M}\right)=\underline{\Xi}\left(m_{\mathcal{A}}, m_{\mathcal{B}}, s\right) \underline{\underline{\pi}}_{G G}\left(m_{\mathcal{A}}, m_{\mathcal{B}}, s\right) .
$$

\section{Proof}

We first note from (4.5) that $\Xi\left(m_{\mathcal{A}}, m_{\mathcal{B}}, s\right)$ is constructed by involving only $\underline{\underline{\sigma}}_{A}(\cdot, s)$ and $\underline{\underline{\sigma}}_{B}(\cdot, s)$, where these matrices are multiplied in the order corresponding to a path connecting $\left[M_{\mathcal{A}}, \overline{\bar{M}}_{\mathcal{B}}\right]$ and $\left[m_{\mathcal{A}}, m_{\mathcal{B}}\right]$, and then the resulting matrices are summed up over all such paths. As can be seen from (3.24) and (3.25), $\underline{\underline{\sigma}}_{A}(\cdot, s)$ corresponds to the joint probability of the first passage time of $\left[J(t), \underline{M}_{n}(t)\right]$ from $\left[i, n_{\mathcal{A}}, n_{\mathcal{B}}\right]$ to $A \cup B$, and this first passage occures at state $\left[j, n_{\mathcal{A}}-1, n_{\mathcal{B}}\right]$. Accordingly, the matrix $\Xi\left(m_{\mathcal{A}}, m_{\mathcal{B}}, s\right)$ is the Laplace transform of a semi-Markov matrix, where its $(i, j)$ element represents the first passage time density of $\underline{M}(t)$ from $\left[M_{\mathcal{A}}, M_{\mathcal{B}}\right]$ to $\left[m_{\mathcal{A}}, m_{\mathcal{B}}\right]$ associated with the state transition of $J(t)$ from $i$ to $j$.

For the process $[J(t), \underline{M}(t)]$ to be at $\left[j, m_{\mathcal{A}}, m_{\mathcal{B}}\right]$ at time $t$ starting with $\left[i, m_{\mathcal{A}}, m_{\mathcal{B}}\right]$, where the Laplace transform of the probability of this event is given by $\beta_{i j}\left(m_{\mathcal{A}}, m_{\mathcal{B}}, s\right)$, it has to first reach a state $\left[r, m_{\mathcal{A}}, m_{\mathcal{B}}\right]$ at time $\tau_{1}<t$ for some $r \in \mathcal{J}$ and the state of $J(t)$ should change from $r$ to $j$ between $\tau_{1}$ and $t$ without having any default at all. The Laplace transform of the probability of the latter event can be described in a matrix form by the right hand side of the expression in the theorem, completing the proof.

Theorem 4.1 together with Theorems 3.1 and 3.2 enables one to compute the time dependent joint probability of $[J(t), \underline{M}(t)]$, which in turn provides a computational vehicle for assessing the pricies of CDO tranches discussed in Section 1, as we show in the subsequent two sections.

\section{Computational Algorithms for Evaluating Time Dependent Joint Probability}

In order to evaluate the risk-neutral premiums of CDO tranches proposed in this paper, the time dependent joint probabilities of $[J(t), \underline{M}(t)]$ have to be computed repeatedly. While Theorem 4.1 in the previous section provides a theoretical foundation for this purpose, applying the result in a straightforward manner may encounter a tremendous computational burden. The purpose of this section is to develop efficient computational algorithms for evaluating the time dependent joint probabilities of $[J(t), \underline{M(t)]}$ by taking advantage of the uniformization procedure of Keilson [7].

We first note from Theorem 4.1 that the matrix Laplace transforms $\underline{\beta}(\underline{m}, s \mid \underline{M})$ of $\underline{\underline{b}}(\underline{m}, t \mid \underline{M})$ can be expressed in terms of $\underline{\underline{\pi}}_{G G}(\underline{m}, s), \underline{\underline{\pi}}_{G A}(\underline{m}, s)$ and $\underline{\pi}_{G B}(\underline{m}, s)$ given in $\overline{\bar{T}}$ Theorem 3.1, as well as $\underline{\underline{\sigma}}_{A}(\underline{m}, s)$ and $\underline{\underline{\sigma}}_{B}(\underline{m}, s)$ in $(3.29)$ and (3.30) respectively. Because of the uniformization procedure employed, all of these matrices belong to a class of matrix Laplace transforms $\mathcal{C}_{\text {LAPL }}$ defined by

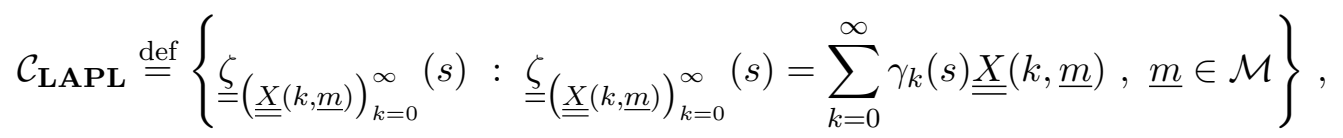

where

$$
\gamma_{k}(s)=\frac{\nu^{k}}{(s+\nu)^{k+1}}
$$

In the real domain, (5.1) and (5.2) can be rewritten as

$$
\mathcal{C}_{\text {REAL }} \stackrel{\text { def }}{=}\left\{\underline{\underline{\mathcal{X}}}(\underline{\underline{X}}(k, \underline{m}))_{k=0}^{\infty}(t): \underline{\underline{\mathcal{X}}}(\underline{\underline{X}}(k, \underline{m}))_{k=0}^{\infty}(t)=\sum_{k=0}^{\infty} g_{k}(t) \underline{\underline{X}}(k, \underline{m}), \underline{m} \in \mathcal{M}\right\}
$$


and

$$
g_{k}(t)=e^{-\nu t} \frac{(\nu t)^{k}}{k !}
$$

respectively. It can be seen from Theorems 3.1 and 3.2 that the five types of matrices $\underline{\underline{\pi}}_{G G}(\underline{m}, s)$, $\underline{\underline{\pi}}_{G A}(\underline{m}, s), \underline{\underline{\pi}}_{G B}(\underline{m}, s), \underline{\underline{\sigma}}_{A}(\underline{m}, s)$ and $\underline{\underline{\sigma}}_{B}(\underline{m}, s)$ belong to $\mathcal{C}_{\mathbf{L A P L}}$.

The fact that the coefficient matrices of a matrix Laplace transform function in $\mathcal{C}_{\text {LAPL }}$ are independent of $s$ and the functional parts are concentrated in the coefficients $\gamma_{k}(s)$ facilitates the matrix convolutions among the matrix functions in $\mathcal{C}_{\text {REAL }}$ greatly, because of a nice property of $\gamma_{k}(s)$ given by

$$
\gamma_{k}(s) \gamma_{\ell}(s)=\frac{1}{\nu} \gamma_{k+\ell+1}(s)
$$

From (5.1) and (5.5), it can be readily seen that $\mathcal{C}_{\text {LAPL }}$ is closed under matrix multiplications. Accordingly, $\mathcal{C}_{\text {REAL }}$ is closed under matrix convolutions. In order to efficiently compute matrix convolutions in $\mathcal{C}_{\text {REAL }}$ by taking advantage of this closeness property, we now introduce the following three mappings involving $\mathcal{C}_{\text {LAPL }}$ and $\mathcal{C}_{\text {REAL }}$.

$$
\text { INV }: \mathcal{C}_{\text {LAPL }} \longrightarrow \mathcal{C}_{\text {REAL }}
$$

with

$$
\underline{\underline{\mathcal{X}}}(\underline{\underline{X}}(k, \underline{m}))_{k=0}^{\infty}(t)=\mathbf{I N V}\left[\underline{\underline{\zeta}}(\underline{\underline{X}}(k, \underline{m}))_{k=0}^{\infty}(s)\right],
$$

$$
\text { MULT }: \mathcal{C}_{\text {LAPL }} \times \mathcal{C}_{\text {LAPL }} \longrightarrow \mathcal{C}_{\text {LAPL }}
$$

with

$$
\begin{aligned}
\underline{\underline{\zeta}}(\underline{\underline{Z}}(r, \underline{m}))_{r=0}^{\infty}(s) & =\mathbf{M U L T}\left[\underline{\underline{\zeta}}(\underline{\underline{X}}(k, \underline{m}))_{k=0}^{\infty}(s), \underline{\underline{\zeta}}(\underline{\underline{Y}}(\ell, \underline{m}))_{\ell=0}^{\infty}(s)\right] \\
& =\underline{\underline{\zeta}}(\underline{\underline{X}}(k, \underline{m}))_{k=0}^{\infty}(s) \times \underline{\underline{\zeta}}(\underline{\underline{Y}}(\ell, \underline{m}))_{n=0}^{\infty}(s)
\end{aligned}
$$

and

$$
\text { SUM }: \mathcal{C}_{\text {LAPL }} \times \mathcal{C}_{\text {LAPL }} \longrightarrow \mathcal{C}_{\text {LAPL }}
$$

with

$$
\begin{aligned}
\underline{\underline{\zeta}}(\underline{\underline{X}}(k, \underline{m})+\underline{\underline{Y}}(k, \underline{m}))_{k=0}^{\infty}(s) & =\mathbf{S U M}\left[\underline{\underline{\zeta}}(\underline{\underline{X}}(k, \underline{m}))_{k=0}^{\infty}(s), \quad \underline{\underline{\zeta}}(\underline{\underline{Y}}(k, \underline{m}))_{k=0}^{\infty}(s)\right] \\
& =\underline{\zeta}(\underline{\underline{X}}(k, \underline{m}))_{k=0}^{\infty}(s)+\underline{\underline{\zeta}}(\underline{\underline{Y}}(\ell, \underline{m}))_{n=0}^{\infty}(s) .
\end{aligned}
$$

Here INV is the matrix inversion operator specifying the matrix function in real domain given a matrix Laplace transform. As the definitions in (5.9) and (5.11) themselves suggest, MULT is the matrix multiplication operator and SUM is the matrix addition operator. From (5.5) and 
(5.9), one observes that

$$
\begin{aligned}
\underline{\underline{\zeta}}(\underline{\underline{Z}}(r, \underline{m}))_{r=0}^{\infty}(s) & =\operatorname{MULT}\left[\underline{\underline{\zeta}}(\underline{\underline{X}}(k, \underline{m}))_{k=0}^{\infty}(s), \underline{\underline{\zeta}}(\underline{\underline{Y}}(\ell, \underline{m}))_{\ell=0}^{\infty}(s)\right] \\
& =\sum_{k=0}^{\infty} \gamma_{k}(s) \underline{\underline{X}}(k, \underline{m}) \sum_{\ell=0}^{\infty} \gamma_{\ell}(s) \underline{\underline{Y}}(\ell, \underline{\underline{m}}) \\
& =\frac{1}{\nu} \sum_{k=0}^{\infty} \sum_{\ell=0}^{\infty} \gamma_{k+\ell+1}(s) \underline{\underline{X}}(k, \underline{m}) \underline{\underline{Y}}(\ell, \underline{m}) \\
& =\frac{1}{\nu} \sum_{r=1}^{\infty} \gamma_{r}(s) \sum_{k+\ell+1=r: k, \ell \geq 0} \underline{\underline{X}}(k, \underline{\underline{m}}) \underline{\underline{Y}}(\ell, \underline{m}) .
\end{aligned}
$$

Consequently, it follows that

$$
\underline{\underline{Z}}(r, \underline{m})=\frac{1}{\nu} \sum_{k+\ell+1=r: k, \ell \geq 0} \underline{\underline{X}}(k, \underline{m}) \underline{\underline{Y}}(\ell, \underline{m}), r=1,2, \cdots .
$$

We are now in a position to summarize computational procedures for evaluating $\underline{b}(\underline{m}, t \mid \underline{M})$. We begin this task by introducing a subroutine named UPDATE as described below. Keeping Figure 4.2 in mind, given the number of corporations $M_{\mathcal{A}}$ and $M_{\mathcal{B}}$ in the industrial sectors $\mathcal{A}$ and $\mathcal{B}$ respectively at time 0 , this subroutine returns $\left(m_{\mathcal{A}}, m_{\mathcal{B}}\right)$ elements which belong to the $n$-th column in the figure, where the resulting matrix is denoted by $\underline{\underline{M}}(n)$ with $\bar{n}$ denoting its column dimension. We note that $0 \leq n \leq M_{\mathcal{A}}+M_{\mathcal{B}}$ and $\underline{\underline{M}}(n)$ is an $\bar{n} \overline{\times 2}$ matrix.

Subroutine 5.1 $(\underline{\underline{M}}(n), \bar{n}) \longleftarrow \operatorname{UPDATE}\left(n, M_{\mathcal{A}}, M_{\mathcal{B}}\right)$

. If $n \leq \min \left(M_{\mathcal{A}}, M_{\mathcal{B}}\right)$, then set $\underline{\underline{M}}(n)=\left[\begin{array}{ll}M_{\mathcal{A}}-n & M_{\mathcal{B}} \\ M_{\mathcal{A}}-n+1 & M_{\mathcal{B}}-1 \\ \vdots & \vdots \\ M_{\mathcal{A}}-1 & M_{\mathcal{B}}-n+1 \\ M_{\mathcal{A}} & M_{\mathcal{B}}-n\end{array}\right]$ and $\bar{n}=n+1$.

. If $\min \left(M_{\mathcal{A}}, M_{\mathcal{B}}\right)<n \leq \max \left(M_{\mathcal{A}}, M_{\mathcal{B}}\right)$, then set

$$
\underline{\underline{M}}(n)=\left[\begin{array}{ll}
n-\min \left(M_{\mathcal{A}}, M_{\mathcal{B}}\right) & \min \left(M_{\mathcal{A}}, M_{\mathcal{B}}\right) \\
n-\min \left(M_{\mathcal{A}}, M_{\mathcal{B}}\right)+1 & \min \left(M_{\mathcal{A}}, M_{\mathcal{B}}\right)-1 \\
\vdots & \vdots \\
n-1 & 1 \\
n & 0
\end{array}\right] \text { and } \bar{n}=\min \left(M_{\mathcal{A}}, M_{\mathcal{B}}\right)+1 .
$$

. If $\max \left(M_{\mathcal{A}}, M_{\mathcal{B}}\right)<n$, then set

$$
\underline{\underline{M}}(n)=\left[\begin{array}{ll}
0 & M_{\mathcal{A}}+M_{\mathcal{B}}-n \\
1 & M_{\mathcal{A}}+M_{\mathcal{B}}-n-1 \\
\vdots & \vdots \\
M_{\mathcal{A}}+M_{\mathcal{B}}-n-1 & 1 \\
M_{\mathcal{A}}+M_{\mathcal{B}}-n & 0
\end{array}\right] \text { and } \bar{n}=M_{\mathcal{A}}+M_{\mathcal{B}}-n+1 .
$$

The main algorithm can then be summarized as follows.

\section{Algorithm 5.2}

Input: 


$$
\begin{aligned}
& \triangleright \mathcal{M}=\left\{0,1, \cdots, M_{\mathcal{A}}\right\} \times\left\{0,1, \cdots, M_{\mathcal{B}}\right\} \\
& \triangleright \mathcal{J}=\{0,1, \cdots, J\} \\
& \triangleright t>0 \\
& \triangleright \nu \text { satisfying }(3.17) \\
& \triangleright \nu_{i}^{+}, i \in \mathcal{J} \backslash\{J\} ; \nu_{i}^{-}, i \in \mathcal{J} \backslash\{0\} \\
& \triangleright \theta_{i j}^{\mathcal{A}}, \theta_{i j}^{\mathcal{B}}, i, j \in \mathcal{J} \\
& \triangleright \rho_{\mathcal{A} \rightarrow \mathcal{A}}, \rho_{\mathcal{B} \rightarrow \mathcal{A}}, \rho_{\mathcal{A} \rightarrow \mathcal{B}}, \rho_{\mathcal{B} \rightarrow \mathcal{B}} \\
& \triangleright \underline{\xi}_{\mathcal{A}}=\left[\xi_{\mathcal{A}}(0), \xi_{\mathcal{A}}(1), \cdots, \xi_{\mathcal{A}}(J)\right]^{\top} \\
& \triangleright \underline{\xi}_{\mathcal{B}}=\left[\xi_{\mathcal{B}}(0), \xi_{\mathcal{B}}(1), \cdots, \xi_{\mathcal{B}}(J)\right]^{\top}
\end{aligned}
$$

Output:

$\triangleleft \underline{\underline{b}}(\underline{m}, t \mid \underline{M})$ for all $\underline{m} \in \mathcal{M}$ where $\underline{M}=\left(M_{\mathcal{A}}, M_{\mathcal{B}}\right)$

Procedure:

1] Compute $\underline{\underline{a}}_{\nu}(\underline{M})$ from (3.18).

2] Based on Theorem 3.2 and (5.1), set $\underline{\underline{\sigma}}_{A}(\underline{M}, s)=\underline{\underline{\zeta}}\left(\nu \underline{\underline{a}}_{\nu: G G}(\underline{M})^{k} \underline{\underline{a}}_{\nu: G A}(\underline{M})\right)_{k=0}^{\infty}(s)$,

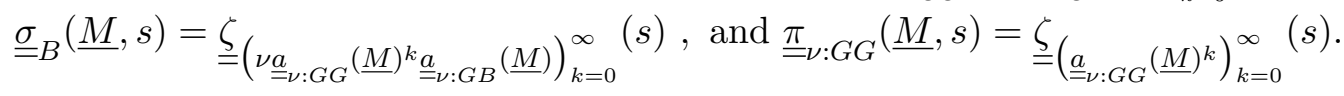

3] $\operatorname{Set} \underline{\underline{\beta}} \underline{\underline{M}}, s \mid \underline{M})=\underline{\pi}_{G G}(\underline{M}, s)$.

4] Compute $\underline{\underline{b}}(\underline{M}, t \mid \underline{M})=\mathbf{I N V}[\underline{\underline{\beta}}(\underline{M}, s \mid \underline{M})]$.

5] Set $n=\ell=1$.

6] LOOP1 : Set $(\underline{\underline{M}}(n), \bar{n}) \longleftarrow \operatorname{UPDATE}\left(n, M_{\mathcal{A}}, M_{\mathcal{B}}\right)$

7] LOOP2 : Set $\underline{m}=\left[m_{\mathcal{A}}, m_{\mathcal{B}}\right] \longleftarrow \quad \ell$-th row of $\underline{\underline{M}}(n)$

8] Compute $\underline{\underline{a}}_{\nu}(\underline{m})$ from (3.18).

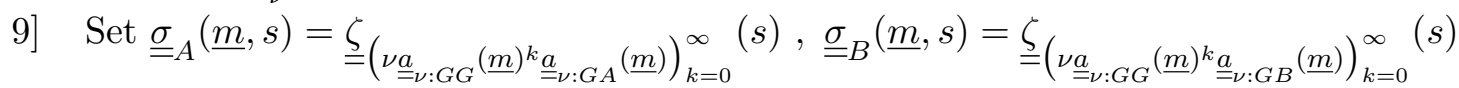



10] Based on (4.4) and (4.5), find

$$
\begin{aligned}
\Xi & \underline{m})=\mathbf{S U M}[\mathbf{M U L T}[ \\
\mathbf{M U L T} & {\left.\left.\left[m_{\mathcal{A}}+1, m_{\mathcal{B}}\right), \underline{\underline{\sigma}}_{A}\left(m_{\mathcal{A}}, m_{\mathcal{B}}+1\right), \underline{\underline{\sigma}}_{B}\left(m_{\mathcal{A}}, m_{\mathcal{B}}+1, s\right)\right]\right] . }
\end{aligned}
$$

11] Set $\underline{\underline{\beta}}(\underline{m}, s \mid \underline{M})=\mathbf{M U L T}\left[\underline{\underline{\Xi}}(\underline{m}), \underline{\underline{\pi}}_{G G}(\underline{m}, s)\right]$.

12] Compute $\underline{\underline{b}}(\underline{m}, t \mid \underline{M})=\mathbf{I N V}[\underline{\underline{\beta}}(\underline{\underline{m}}, s \mid \underline{M})]$.

$13] \longrightarrow(\bar{n} \geq \ell \leftarrow \ell+1) /$ LOOP2

14] Set $\ell=1$.

15] $\longrightarrow\left(M_{\mathcal{A}}+M_{\mathcal{B}} \geq n \leftarrow n+1\right) /$ LOOP1

16] Compute $\underline{\underline{\beta}}(\underline{0}, s \mid \underline{M})=\mathbf{M U L T}\left[\underline{\Xi}(\underline{0}), \underline{\underline{\pi}}_{G G}(\underline{0}, s)\right]$.

17] Compute $\underline{\underline{b}}(\underline{0}, t \mid \underline{M})=\mathbf{I N V}[\underline{\underline{\beta}}(\underline{0}, s \mid \underline{M})]$.

18] END.

A few remarks are worth noting. 
Remark 5.3 a) Given $t>0$, the sequence $\left(g_{k}(t)\right)_{k=0}^{\infty}$ is unimodal with the peak shifting to the right as $t$ becomes larger. In order to secure a truncation accuracy, say $\varepsilon>0$, it is necessary to pick up terms which are grater than $\varepsilon$ centered at the peak.

b) Keeping the point in a) above in mind, the vector values $\left[g_{k+1}\left(t_{1}\right), g_{k+1}\left(t_{2}\right), \cdots, g_{k+1}\left(t_{N}\right)\right]$ can be generated recursively from $\left[g_{k}\left(t_{1}\right), g_{k}\left(t_{2}\right), \cdots, g_{k}\left(t_{N}\right)\right]$.

\section{Evaluation of CDO Premiums and Prices of CDO Option of European Type}

In order to facilitate the necessary computations for evaluating the premium $c_{\left[K_{a}, K_{d}\right]}^{*}$ of the CDO Tranche $\left[K_{a}, K_{d}\right]$ discussed in Section 1, we first assess the joint probability distribution of the number of corporations having defaulted by time $t$ in the industrial sector $\mathcal{A}$ and that in the industrial sector $\mathcal{B}$. Assuming that the macro economic condition is in state $i$ at time 0 , i.e. $J(0)=i$, it can be readily seen that

$$
\begin{aligned}
& \mathbb{P}\left\{D_{\mathcal{A}}(t)=d_{\mathcal{A}}, D_{\mathcal{B}}(t)=d_{\mathcal{B}} \mid J(0)=i\right\} \\
= & \mathbb{P}\left\{M_{\mathcal{A}}(t)=M_{\mathcal{A}}-d_{\mathcal{A}}, M_{\mathcal{B}}(t)=M_{\mathcal{B}}-d_{\mathcal{B}} \mid J(0)=i\right\} \\
= & \sum_{j \in \mathcal{J}} b_{i j}\left(M_{\mathcal{A}}-d_{\mathcal{A}}, M_{\mathcal{B}}-d_{\mathcal{B}}, t \mid \underline{M}\right),
\end{aligned}
$$

which can be computed by Algorithm 5.2.

We next turn our attention to evaluate $\mathbb{E}\left[L_{\left[K_{a}, K_{d}\right]}(t) \mid J(0)=i\right]$. The set $\left\{\left(d_{\mathcal{A}}, d_{\mathcal{A}}\right): 0 \leq d_{\mathcal{A}} \leq\right.$ $\left.M_{\mathcal{A}}, 0 \leq d_{\mathcal{B}} \leq M_{\mathcal{B}}\right\}$ can be decomposed into three regions $\mathcal{D}_{\mathrm{I}}, \mathcal{D}_{\text {II }}$ and $\mathcal{D}_{\text {III }}$ as follows.

$$
\begin{aligned}
\mathcal{D}_{\mathrm{I}} & =\left\{\left(d_{\mathcal{A}}, d_{\mathcal{B}}\right): 0 \leq d_{\mathcal{A}} \leq \frac{K_{a}-N_{\mathcal{B}}\left(1-R_{\mathcal{B}}\right)}{N_{\mathcal{A}}\left(1-R_{\mathcal{A}}\right)} d_{\mathcal{B}}\right\} \\
\mathcal{D}_{\mathrm{II}} & =\left\{\left(d_{\mathcal{A}}, d_{\mathcal{B}}\right): \frac{K_{a}-N_{\mathcal{B}}\left(1-R_{\mathcal{B}}\right)}{N_{\mathcal{A}}\left(1-R_{\mathcal{A}}\right)} d_{\mathcal{B}} \leq d_{\mathcal{A}} \leq \frac{K_{d}-N_{\mathcal{B}}\left(1-R_{\mathcal{B}}\right)}{N_{\mathcal{A}}\left(1-R_{\mathcal{A}}\right)} d_{\mathcal{B}}\right\} \\
\mathcal{D}_{\mathrm{III}} & =\left\{\left(d_{\mathcal{A}}, d_{\mathcal{B}}\right): d_{\mathcal{A}} \geq \frac{K_{d}-N_{\mathcal{B}}\left(1-R_{\mathcal{B}}\right)}{N_{\mathcal{A}}\left(1-R_{\mathcal{A}}\right)} d_{\mathcal{B}}\right\}
\end{aligned}
$$

From (1.3), one then sees that

$$
L_{\left[K_{a}, K_{d}\right]}(t)=\left\{\begin{array}{ll}
0 & \text { if }\left(D_{\mathcal{A}}(t), D_{\mathcal{B}}(t)\right) \in \mathcal{D}_{\mathrm{I}} \\
l(t)-K_{a} & \text { if }\left(D_{\mathcal{A}}(t), D_{\mathcal{B}}(t)\right) \in \mathcal{D}_{\mathrm{II}} \\
K_{d}-K_{a} & \text { if }\left(D_{\mathcal{A}}(t), D_{\mathcal{B}}(t)\right) \in \mathcal{D}_{\mathrm{III}}
\end{array} .\right.
$$

It then follows from $(1.11)$ that, with $\underline{M}=\left(\mathcal{M}_{\mathcal{A}}, \mathcal{M}_{\mathcal{B}}\right)$ and $\underline{d}=\left(d_{\mathcal{A}}, d_{\mathcal{B}}\right)$, one has

$$
\begin{aligned}
\mathbb{E}\left[L_{\left[K_{a}, K_{d}\right]}(t) \mid J(0)=i\right] & =\sum_{\underline{d} \in \mathcal{D}_{\text {II }}} \sum_{j \in \mathcal{J}}\left(l(t)-K_{a}\right) b_{i j}(\underline{M}-\underline{d}, t \mid \underline{M}) \\
& +\left(K_{d}-K_{a}\right) \sum_{\underline{d} \in \mathcal{D}_{\text {III }}} \sum_{j \in \mathcal{J}} b_{i j}(\underline{M}-\underline{d}, t \mid \underline{M}) .
\end{aligned}
$$

Consequently, the CDO premium $c_{\left[K_{a}, K_{d}\right]}^{*}$ of main interest can be obtained from (1.7) and (6.3) based on Algorithm 5.2.

The CDO option of European type introduced in Section 1 is concerned with the decision at time 0 of whether or not to buy an option for entering into a CDO contract commencing at time $\tau_{0}>0$. Clearly, the value of the option depends on the width of the distribution of the CDO value at time $\tau_{0}$. In order to assess the option value specified in (1.9), let $D_{P R T}\left(\tau_{0} \mid j\right)$ and $D_{P R M}\left(\tau_{0}, \tilde{c}_{\left[K_{a}, K_{d}\right]} \mid j\right)$ be the protectionleg and premiumleg at time $\tau_{0}$ given that $J\left(\tau_{0}\right)=j$, where 
$\tilde{c}_{\left[K_{a}, K_{d}\right]}$ is the agreed premium which is not necessarily at equilibrium. More formally, we define

$$
\begin{aligned}
& =\sum_{k=0}^{K} e^{-r \tau_{k}} \mathbb{E}\left[L_{\left[K_{a}, K_{d}\right]}\left(\tau_{k}\right)-L_{\left[K_{a}, K_{d}\right]}\left(\tau_{k-1}\right) \mid J\left(\tau_{0}\right)=j\right], \\
(6.5) D_{P R M}\left(\tau_{0}, \tilde{c}_{\left[K_{a}, K_{d}\right]} \mid j\right) & =\sum_{k=0}^{K} e^{-r \tau_{k}} \mathbb{E}\left[P A Y_{\text {buy } \rightarrow \text { sell }}\left(\tilde{c}_{\left[K_{a}, K_{d}\right]}, \tau_{k}\right) \mid J\left(\tau_{0}\right)=j\right] \\
& =\tilde{c}_{\left[K_{a}, K_{d}\right]} \cdot \sum_{k=0}^{K} e^{-r \tau_{k}} \mathbb{E}\left[\left(\left(K_{d}-K_{a}\right)-L_{\left[K_{a}, K_{d}\right]}\left(\tau_{k}\right)\right) \mid J\left(\tau_{0}\right)=j\right] .
\end{aligned}
$$

Then the option value at time 0 in (1.9) is obtained by unconditioning the difference of $D_{P R T}\left(\tau_{0} \mid j\right)$ and $D_{P R M}\left(\tau_{0}, \tilde{c}_{\left[K_{a}, K_{d}\right]} \mid j\right)$ in (6.4) and (6.5) respectively. Namely, one has

$$
e^{-r \tau_{0}} \sum_{j=0}^{J} \mathbb{P}\left\{J\left(\tau_{0}\right)=j\right\} \cdot \max \left\{D_{P R T}\left(\tau_{0} \mid j\right)-D_{P R M}\left(\tau_{0}, \tilde{c}_{\left[K_{a}, K_{d}\right]} \mid j\right), 0\right\},
$$

where $J\left(\tau_{0}\right)$ can be computed by applying Keilson's uniformization procedure described in Section 3 to the marginal process $J(t)$.

\section{Numerical Results}

In this section, we demonstrate that the numerical algorithms developed in Sections 3 through 5 can be implemented with speed and accuracy. For this purpose, we suppose that the industrial sector $\mathcal{A}$ consists of a group of manufacturers producing consumer products and let the industrial sector $\mathcal{B}$ be the associated Tier 1 suppliers. It is assumed that the industrial sector $\mathcal{A}$ has 40 corporations while the industrial sector $\mathcal{B}$ contains 60 corporations.

The stochastic process $J(t)$ describing the macro economic conditions is assumed to follow the Ehrenfest process on $\mathcal{J}=\{0,1, \cdots, 2 V\}$. Here, a higher state implies a better economic condition with state $\mathrm{V}$ corresponding to a normal economic condition. The Ehrenfest process has the upward transition rate $\nu_{i}^{+}$and the downward transition rate $\nu_{i}^{-}$at state $i \in \mathcal{J}$ given by

$$
\nu_{i}^{+}=v \cdot\left(V-\frac{i}{2}\right) ; \nu_{i}^{-}=v \cdot \frac{i}{2},
$$

where $v$ is a positive constant. It should be noted that $\nu_{i}^{-}$dominates $\nu_{i}^{+}$for $V<i \leq 2 V$ while this dominance is reversed for $0 \leq i<V$. The Ehrenfest process is chosen because of this dynamics. That is, as the process deviates from the normal economic condition, the driving force to bring the process back to it becomes stronger, see e.g. Sumita et al.(2003).

Given the macro economic condition $i \in \mathcal{J}$, the default intensities $\xi_{\mathcal{A}}(i)$ and $\xi_{\mathcal{B}}(i)$ are given by

$$
\xi_{\mathcal{A}}(i)=\alpha_{\mathcal{A}} e^{-\beta_{\mathcal{A}}(i-V)} ; \xi_{\mathcal{B}}(i)=\alpha_{\mathcal{B}} e^{-\beta_{\mathcal{B}}(i-V)} .
$$

As for the linear coefficients of the default intensities, which are proportional to the number of defaulted corporations, we consider the following four cases.

$$
\begin{array}{ll}
(\text { Case } 1) & \rho_{\mathcal{A} \rightarrow \mathcal{A}}=\rho_{\mathcal{B} \rightarrow \mathcal{B}}=\rho_{\mathcal{A} \rightarrow \mathcal{B}}=\rho_{\mathcal{B} \rightarrow \mathcal{A}}=0 \\
(\text { Case } 2) & \rho_{\mathcal{A} \rightarrow \mathcal{A}}=\rho_{\mathcal{B} \rightarrow \mathcal{B}}>0, \rho_{\mathcal{A} \rightarrow \mathcal{B}}=\rho_{\mathcal{B} \rightarrow \mathcal{A}}=0 \\
(\text { Case } 3) & \rho_{\mathcal{A} \rightarrow \mathcal{A}}=\rho_{\mathcal{B} \rightarrow \mathcal{B}}>\rho_{\mathcal{A} \rightarrow \mathcal{B}}>0, \rho_{\mathcal{B} \rightarrow \mathcal{A}}=0 \\
\text { (Case } 4) & 0<\rho_{\mathcal{A} \rightarrow \mathcal{A}}=\rho_{\mathcal{B} \rightarrow \mathcal{B}}<\rho_{\mathcal{A} \rightarrow \mathcal{B}}, \rho_{\mathcal{B} \rightarrow \mathcal{A}}=0
\end{array}
$$


Case 1 implies that active corporations are not affected by the past defaults at all. In Case 2, such interactions occur only within the same industrial sectors, where the influencing power of a defaulted corporation against the still active corporations would be the same for the two industrial sectors $\mathcal{A}$ and $\mathcal{B}$. Case 3 assumes that the interactions between active corporations and defaulted corporations within the same industrial sectors would be as for Case 2, but in addition, a default in the industrial sector $\mathcal{A}$ would weakly affect the active Tier 1 suppliers in the industrial sector $\mathcal{B}$. The situation in Case 4 is similar to Case 3 except that the influencing power of a defaulted manufacturer in $\mathcal{A}$ against the active Tier 1 suppliers in $\mathcal{B}$ would be stronger than the influencing power within each industrial sector.

The basic set of the underlying parameter values are summarized in Tables 7.1 and 7.2, which would be employed throughout the section unless specified otherwise. Since the Tier 1 suppliers in $\mathcal{B}$ are likely to be more responsive to the macro economic condition than the manufactures in $\mathcal{A}$, we set $\alpha_{\mathcal{A}}=0.01<0.04=\alpha_{\mathcal{B}}$ based on (7.2), while the exponential factors $\beta_{\mathcal{A}}$ and $\beta_{\mathcal{B}}$ are assumed to be equal with $\beta_{\mathcal{A}}=\beta_{\mathcal{B}}=0.3$. By the same reason, the default probability upon transition of the macro economic condition for the industrial sector $\mathcal{B}$ is higher than that for the industrial sector $\mathcal{A}$. As for the recovery rate, the basic model assumes $R_{\mathcal{A}}=0.4$ while $R_{\mathcal{B}}=0.3$. The tranche of basic model is $\left[K_{a}, K_{d}\right]=[0.05,0.15]$, and the interest rate is set to be $r=0.01$.

\begin{tabular}{lllll}
\hline$J=6(V=3)$ & $\alpha_{\mathcal{A}}=0.01$ & $\alpha_{\mathcal{B}}=0.04$ & $K_{a}=0.05$ & $R_{\mathcal{A}}=0.4$ \\
$v=3.0$ & $\beta_{\mathcal{A}}=0.3$ & $\beta_{\mathcal{B}}=0.3$ & $K_{d}=0.15$ & $R_{\mathcal{B}}=0.3$ \\
$r=0.01$ & $\Theta_{i j}^{\mathcal{A}}=0.048(\forall i, j \in \mathcal{J})$ & $\Theta_{i j}^{\mathcal{B}}=0.078(\forall i, j \in \mathcal{J})$ & $N_{\mathcal{A}}=N_{\mathcal{B}}=0.01$ & \\
\hline
\end{tabular}

Table 7.1: Basic Set of Parameter Values

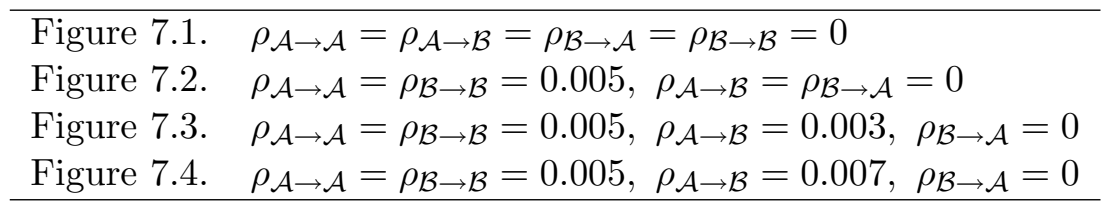

Table 7.2: Linear Coefficients of Default Intensities

Figures 7.1 through 7.4 depict the joint distribution of $\left[D_{\mathcal{A}}(t), D_{\mathcal{B}}(t)\right]$ at the maturity of the CDO at time $t=3$ years for Cases 1 through 4 . For Case 1 , defaults are not contagious and both the number of corporations defaulted by time $t$ in $\mathcal{A}$ and that in $\mathcal{B}$ are contained within a relatively small region with $\mathbb{E}\left(D_{\mathcal{A}}(3)\right)=2.55$ and $\mathbb{E}\left(D_{\mathcal{B}}(3)\right)=9.16$. The spread of the joint distribution increases as interactions between defaults are introduced for Cases 2 through 4 . It can be seen that the industrial sector $\mathcal{A}$ suffers less from such interactions, where the mean hardly changes and stays around 3.38 while the variance increases from 2.50 for Case 1 to about 6.2 for Cases 2 through 4 . The Tier 1 suppliers in the industrial sector $\mathcal{B}$ are more fragile, with the mean increasing from 9.16 for Case 1, 13.54 for Case 2 and 14.37 for Case 3 to 15.44 for Case 4, as well as the variance rapidly growing from 9.15 for Case 1, 43.41 for Case 2 and 52.32 for Case 3 to 66.84 for Case 4.

Figures 7.5 through 7.12 plot the values of the premium of a CDO for Cases 1 through 4 respectively. Three different curves in each figure correspond to the combinations of the changes of the ratio of the bad debt per default in $\mathcal{A}$ denoted by $B D_{\mathcal{A}}$ v.s. that in $\mathcal{B}$ denoted by $B D_{\mathcal{B}}$, as well as the changes of the recovery rate in $\mathcal{B}$, as summarized in Table 7.3. More specifically, Figures 7.5 and 7.6 correspond to Cases 1-a and 1-b, Figures 7.7 and 7.8 represent Cases 2-a and 2-b, Figures 7.9 and 7.10 illustrate Cases 3-a and 3-b, and finally Figures 7.11 and 7.12 depict Cases 4 -a and 4-b. The horizontal axis represents the shift of the tranche specified by

$$
\left[K_{a}, K_{d}\right]=[0.05+k \times 0.01,0.15+k \times 0.01], k=0,1, \cdots, 20 .
$$




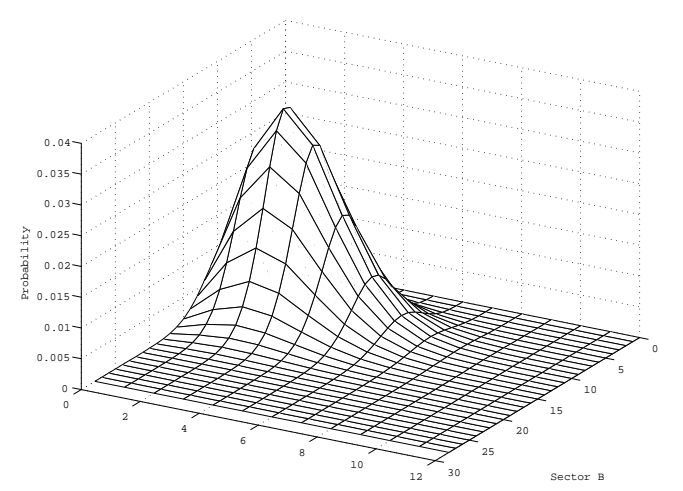

Figure 7.1: Case1

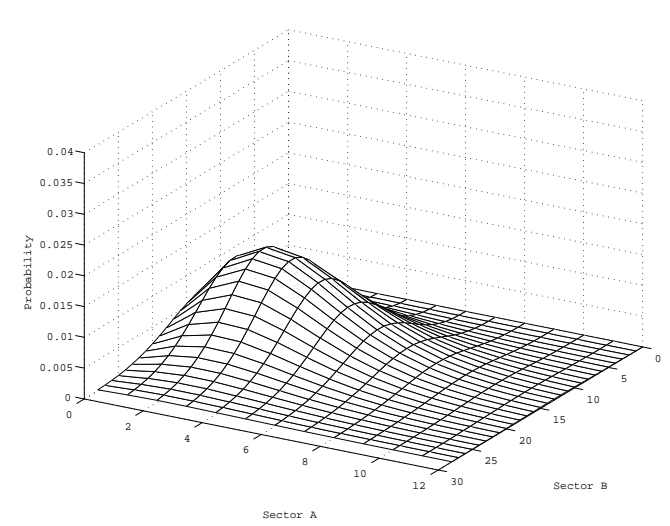

Figure 7.2: Case2

$$
\begin{aligned}
& \mathbb{E}\left(D_{\mathcal{A}}(3)\right)=2.54576 \\
& \mathbb{E}\left(D_{\mathcal{B}}(3)\right)=9.15637 \\
& \mathbb{V}\left(D_{\mathcal{A}}(3)\right)=2.50279 \\
& \mathbb{V}\left(D_{\mathcal{B}}(3)\right)=9.15462 \\
& \operatorname{Cov}\left(D_{\mathcal{A}}(3), D_{\mathcal{B}}(3)\right)=21.37221
\end{aligned}
$$

\begin{tabular}{l}
$\mathbb{E}\left(D_{\mathcal{A}}(3)\right)=3.38355$ \\
$\mathbb{E}\left(D_{\mathcal{B}}(3)\right)=13.54370$ \\
$\mathbb{V}\left(D_{\mathcal{A}}(3)\right)=6.21921$ \\
$\mathbb{V}\left(D_{\mathcal{B}}(3)\right)=43.40890$ \\
$\operatorname{Cov}\left(D_{\mathcal{A}}(3), D_{\mathcal{B}}(3)\right)=20.63292$ \\
\hline
\end{tabular}

ne observes that the CDO premium decreases as $k$ increases. We recall that the protection seller would start paying to the protection buyer when the cumulative loss exceeds the attachment point $K_{a}$. The maximum possible payment by the protection seller to the protection buyer is given by the width of the tranche $K_{d}-K_{a}$. In our example, as can be seen from (7.3), $K_{a}$ increases as $k$ increases while $K_{d}-K_{a}$ remains constant. Accordingly, the risk for the protection seller consistently decreases as $k$ increases, resulting in the decrease of the CDO premium. For each of (i-a) through (iii-b), the corresponding CDO premium increases from Case 1 to Case 4. This is also plausible because the default intensities increase from Case 1 to Case 4 and hence the risk for the protection seller increases accordingly.

\begin{tabular}{llll}
\hline i-a) & $B D_{\mathcal{A}}: B D_{\mathcal{B}}=1: 1, R_{\mathcal{B}}=0.3$ & i-b) & $B D_{\mathcal{A}}: B D_{\mathcal{B}}=2: 1, R_{\mathcal{B}}=0.3$ \\
ii-a) & $B D_{\mathcal{A}}: B D_{\mathcal{B}}=1: 1, R_{\mathcal{B}}=0.4$ & ii-b) & $B D_{\mathcal{A}}: B D_{\mathcal{B}}=2: 1, R_{\mathcal{B}}=0.4$ \\
iii-a) & $B D_{\mathcal{A}}: B D_{\mathcal{B}}=1: 1, R_{\mathcal{B}}=0.5$ & iii-b) & $B D_{\mathcal{A}}: B D_{\mathcal{B}}=2: 1, R_{\mathcal{B}}=0.5$ \\
\hline
\end{tabular}

Table 7.3: Bad Debt per Default and Recovery Rate for Figures 7.5 through 7.12.

In Figures 7.13 through 7.16, the prices of the CDO option of European type, from the point of view of the protection buyer, are exhibited for Cases 1 through 4 respectively. It is assumed that the option is defined on a CDO commencing at $\tau_{0}=1$ and maturing at $\tau_{K}=3$. The horizontal axis scales the ratio between the agreed premium $\tilde{c}_{\left[K_{a}, K_{d}\right]}$ and the theoretical equilibrium premium $c_{\left[K_{a}, K_{d}\right]}^{*}$. For each figure, four curves (i) through (iv) are plotted by varying the attachment point $K_{a}$ and the detachment point $K_{d}$ while holding the width of tranche intact, as specified in Table 7.4. As the agreed premium $\tilde{c}_{\left[K_{a}, K_{d}\right]}$ increases, the payment from the protection buyer to the protection seller increases. Accordingly, the option value decreases as $\tilde{c}_{\left[K_{a}, K_{d}\right]} / c_{\left[K_{a}, K_{d}\right]}^{*}$ increases 


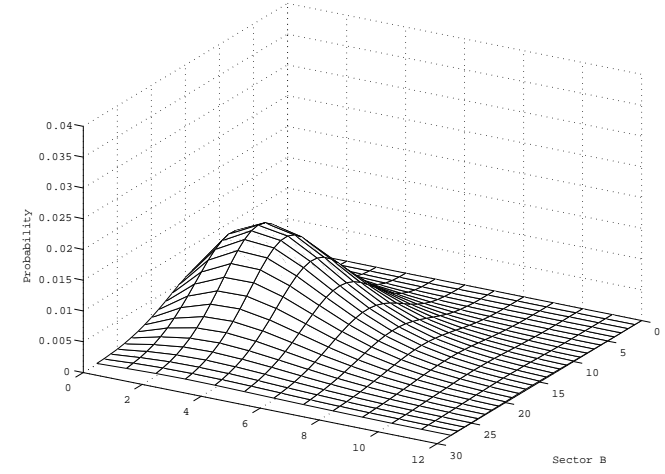

Figure 7.3: Case3

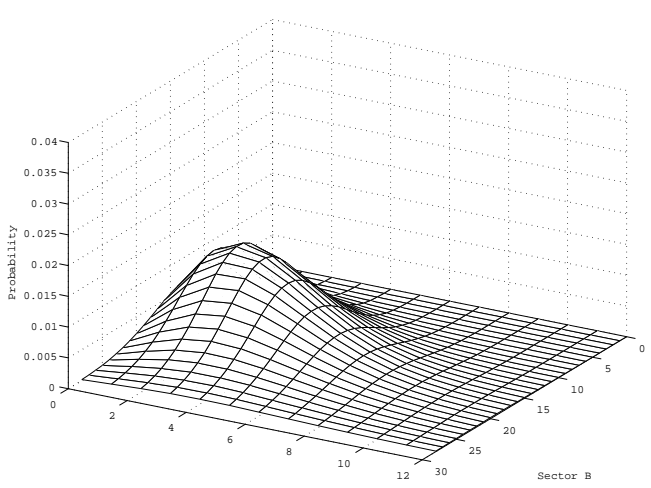

Figure 7.4: Case4

$$
\begin{aligned}
& \mathbb{E}\left(D_{\mathcal{A}}(3)\right)=3.38355 \\
& \mathbb{E}\left(D_{\mathcal{B}}(3)\right)=14.37336 \\
& \mathbb{V}\left(D_{\mathcal{A}}(3)\right)=6.21921 \\
& \mathbb{V}\left(D_{\mathcal{B}}(3)\right)=52.31569 \\
& \operatorname{Cov}\left(D_{\mathcal{A}}(3), D_{\mathcal{B}}(3)\right)=20.63568
\end{aligned}
$$

$$
\begin{aligned}
& \mathbb{E}\left(D_{\mathcal{A}}(3)\right)=3.38351 \\
& \mathbb{E}\left(D_{\mathcal{B}}(3)\right)=15.43703 \\
& \mathbb{V}\left(D_{\mathcal{A}}(3)\right)=6.21905 \\
& \mathbb{V}\left(D_{\mathcal{B}}(3)\right)=66.83824 \\
& \operatorname{Cov}\left(D_{\mathcal{A}}(3), D_{\mathcal{B}}(3)\right)=20.63750
\end{aligned}
$$

along the horizontal axis. For each of (i) through (iv), the corresponding CDO option prices increases from Case 1 to Case 4, since the default intensities increase from Case 1 to Case 4 and hence the risk for the protection buyer (independent of the CDO) increases accordingly. As the tranche shifts to the right while holding the width of tranche intact, the CDO premium decreases while the probability of a range, to which the premium is applied at each installment, being greater than $x$ increases. The former overwhelms the latter, and the option value decreases from (i) to (iv).

\begin{tabular}{ll}
{$\left[K_{a}, K_{d}\right]$} & $=[0.05+k \times 0.01,0.15+k \times 0.01]$ \\
\hline (i) & {$\left[K_{a}, K_{d}\right]=[0.05,0.15]$ with $k=0$} \\
(ii) & {$\left[K_{a}, K_{d}\right]=[0.08,0.18]$ with $k=3$} \\
(iii) & {$\left[K_{a}, K_{d}\right]=[0.11,0.21]$ with $k=6$} \\
(iv) & {$\left[K_{a}, K_{d}\right]=[0.14,0.24]$ with $k=9$} \\
\hline
\end{tabular}

Table 7.4: Underlying Tranches for Figures 7.13 through 7.16.

\section{Concluding Remarks}

In this paper, a credit risk model is considered, with two industrial sectors $\mathcal{A}$ and $\mathcal{B}$ having $M_{\mathcal{A}}$ and $M_{\mathcal{B}}$ active corporations respectively at time $t=0$. A two-layer Markov chain model is developed for capturing the effects of the macro economic condition on individual defaults, as well as the impact of defaults on performance of active corporations. The macro economic condition is described as a birth-death process, while another Markov chain represents the stochastic characteristics of 


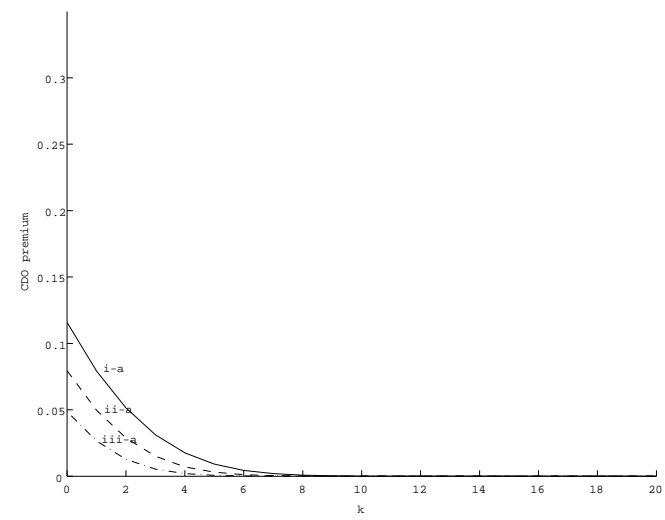

Figure 7.5: Case 1-a

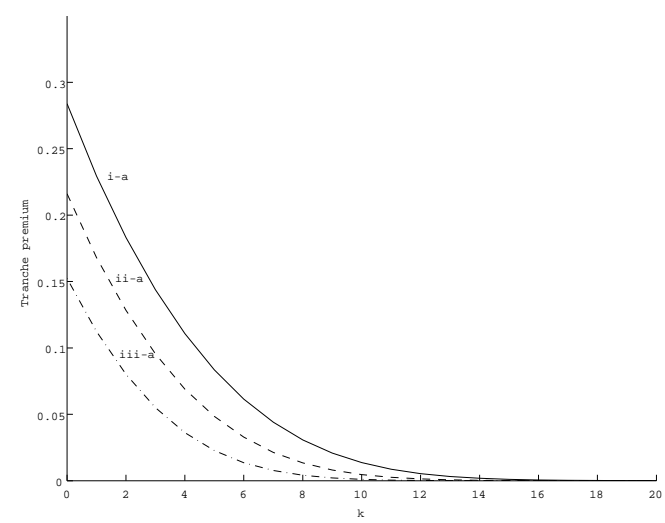

Figure 7.7: Case 2-a

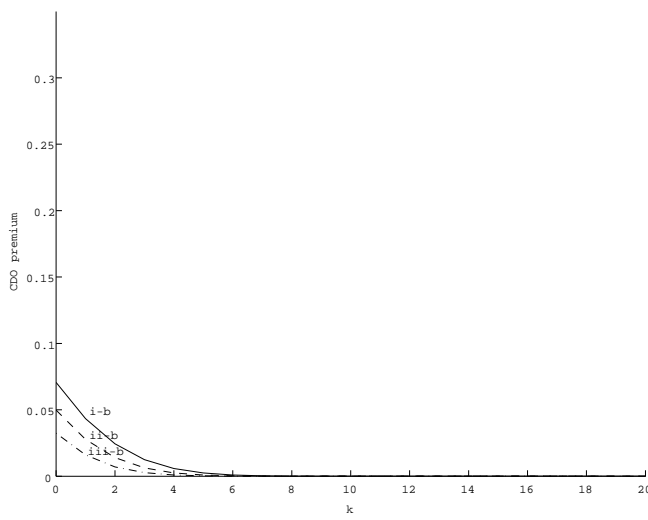

Figure 7.6: Case 1-b

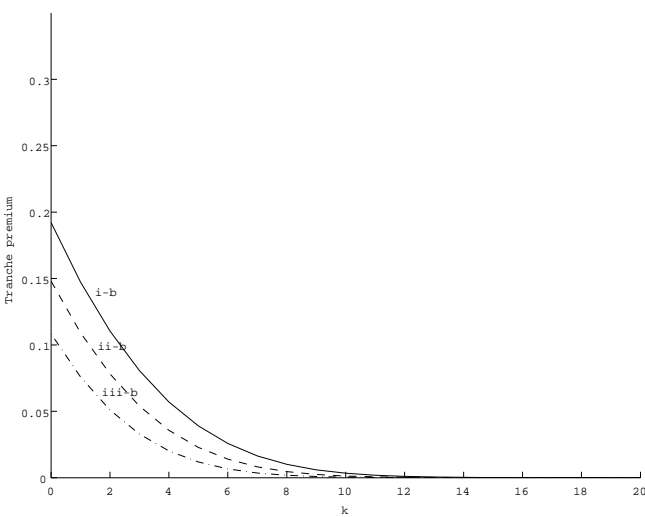

Figure 7.8: Case 2-b

defaults with default intensities dependent on the state of the birth-death process, the number of defaults in $\mathcal{A}$ and that in $\mathcal{B}$ by time $t$. Although the state space of the two-layer Markov chain is huge, the fundamental absorbing trivariate process with a reasonable state space size could capture the first passage time structure of the two-layer Markov chain, thereby enabling one to evaluate the joint probability of the number of defaults in $\mathcal{A}$ and that in $\mathcal{B}$ by time $t$ via the uniformization procedure of Keilson with speed and accuracy. This in turn enables one to value the equilibrium premiums of CDOs defined on the underlying credit portfolio and the prices of the related CDO options of European type. Some numerical examples are presented for illustrating the efficiency of the computational procedures developed in the paper.

\section{References}

[1] Azizpour, S., Giesecke, K. 2008. Self-Exciting corporate defaults: contagion vs. frailty. Working paper, Stanford University.

[2] Collin-Dufresne, P., Goldstein, R., Helwege, J. 2003. Is credit event risk proced? Modeling contagion via the updating of beliefs.

Working paper, Haas School, University of California, Berkely.

[3] Das, S. R., Duffie, D., Kapadia, N., Saita, L. 2007. Common failings: How corporate defaults are correlated.

Journal of Finance 62 93-117.

[4] Frey, R., Backhaus, J. 2006. Portfolio credit risk models with interacting default intensities: a Markovian approach. 


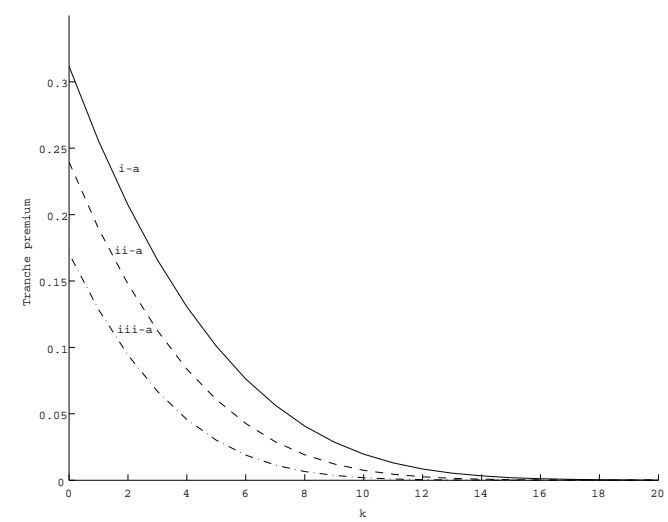

Figure 7.9: Case 3-a

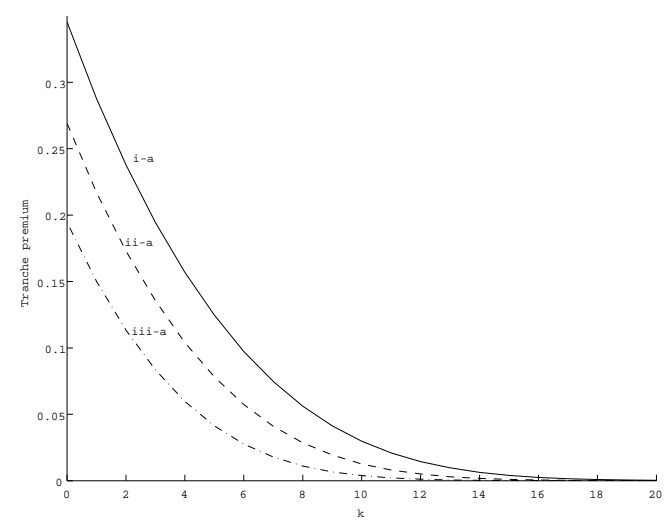

Figure 7.11: Case 4-a

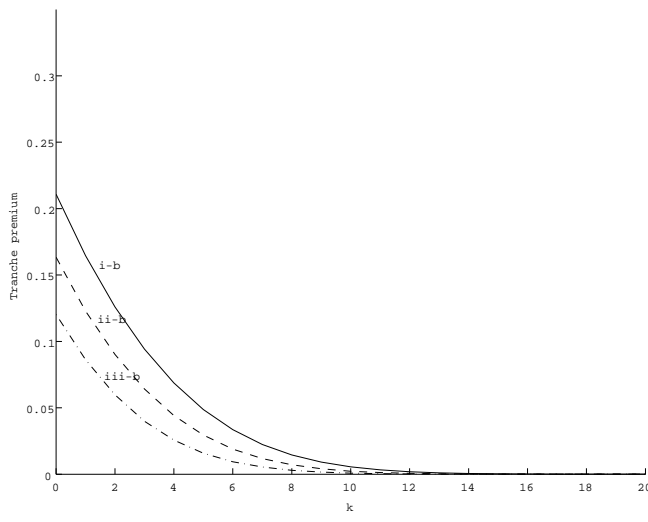

Figure 7.10: Case 3-b



Figure 7.12: Case 4-b

Working paper, Department of Mathematics. University of Leipzig.

[5] Herbertsson, A. Rootzen, H. 2008. Pricing kth-to-default swaps under default contagion:the matrixanalytic approach.

The Journal of Computational Finance 12(1), 1-30.

[6] Jarrow, L., Yu, F. 2001. Counterparty risk and the pricing of defaultable securities. Journal of Finance 56 1765-1800.

[7] Keilson, J. 1979. Markov Chain Models: Rarity and Exponentiality. Applied Mathematical Science Series, No.28, Springer, New York.

[8] O'Kane, D. 2008. Modelling single-name and multi-name credit derivatives. Wiley Finance.

[9] Sumita, U., Gotoh, J., Jin, H. 2003. Numerical exploration of dynamic behavior of the Ornstein-Unlenbeck process via Ehrenfest process approximation.

Applied Probability Trust 11

[10] Yu, F. 2007. Correlated defaults in intensity-based models. Mathematical Finance 17(2) 155-173.

[11] Ding, X., Giesecke, K., Tomecek, P. 2008. Time-changed birth processes and multi-name credit derivatives.

Forthcoming in Operations Research.

[12] Zheng, H., Jiang, L. 2009. Basket CDS pricing with interacting intensities.

Finance and Stochastics 13 445-469. 


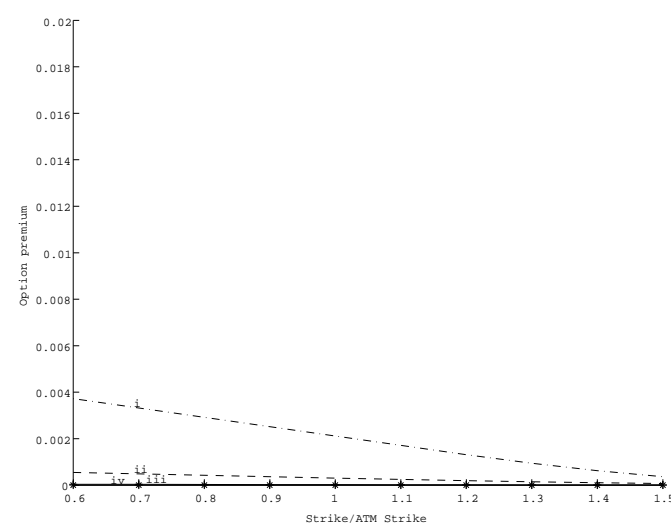

Figure 7.13: Case 1

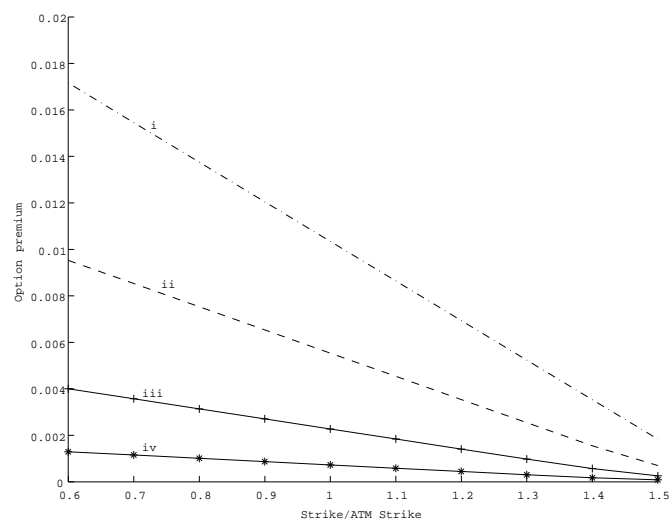

Figure 7.15: Case 3

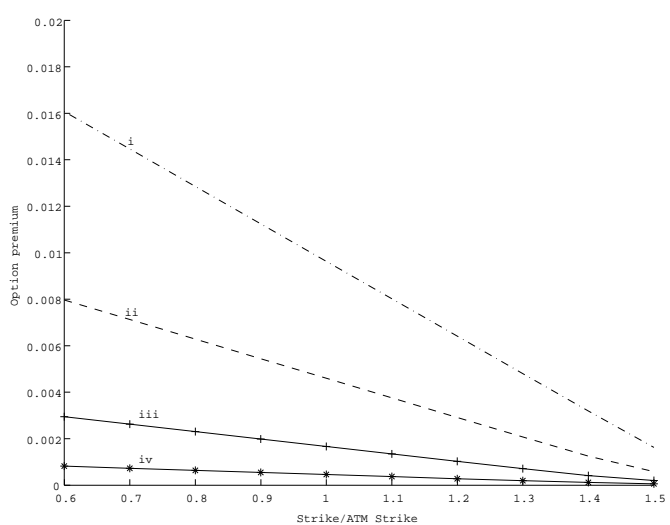

Figure 7.14: Case 2

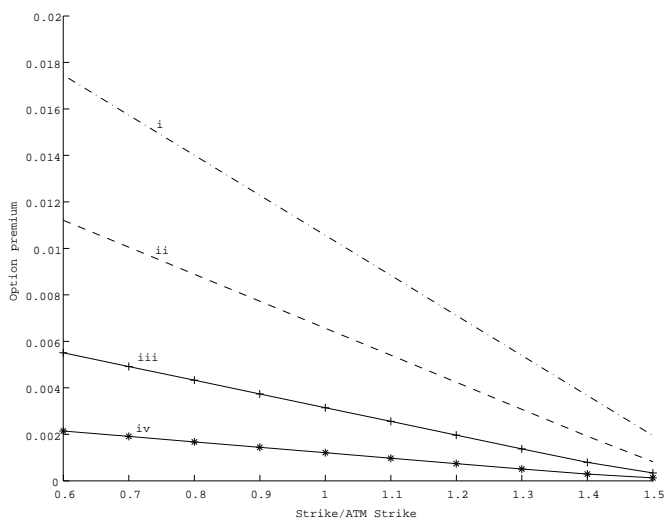

Figure 7.16: Case 4 